\title{
HUMAN MEIOSIS VII. CHIASMA FORMATION IN HUMAN SPERMATOCYTES
}

by

PREBEN BACH HOLM

and

SØREN WILKEN RASMUSSEN

Department of Physiology, Carlsberg Laboratory

Gamle Carlsberg Vej 10, DK-2500 Copenhagen Valby

Keywords: Synaptonemal complex, recombination nodule, bar, XY bivalent, kinetochore, centriole, terminalization.

The diplotene and diakinesis stages in human spermatocytes have been analyzed by serial sectioning and three dimensional reconstruction of 17 nuclei covering the period from early diplotene to prometaphase $I$. The analysis has permitted the following observations and conclusions: 1) The diplotene and diakinesis stages in human spermatocytes are very short but can be subdivided into early, mid and late substages on the basis of changes in nuclear shape, centriolar behaviour, synaptonemal complex elimination and bivalent contraction. The ultrastructure of the autosomal bivalents and the XY bivalent is described. 2) Synaptonemal complex elimination is initiated at a low number of sites at early diplotene, creating about 70 remaining stretches of synaptonemal complex. Degradation continues during diplotene but is confined to the ends of these segments as the total number of distinct segments is unchanged during diplotene. The distribution of the segments among and along the bivalents is similar to that of crossovers and chiasmata, suggesting that the crossovers are contained in these segments. In virtually all $X Y$ bivalents, a short synaptonemal complex segment combines the homologous regions of the $\mathrm{X}$ and the $\mathrm{Y}$ chromosome. 3) The length of the synaptonemal complex segments remains unchanged from early diakinesis until metaphase $\mathrm{I}$. The mean number of segments at early-mid diakinesis amounts to 62 . At late diakinesis the segments are eliminated and replaced by chromatin bridges, the eliminated segments forming small polycomplexes. By metaphase I these polycomplexes are shed from the bivalents. 5) A comparison between the chiasma distribution as seen in the light microscope and that of crossovers or synaptonemal complex segments at mid-late diplotene reveal that chiasmata occupy more distal positions. 6) It is proposed that the pattern of elimination of the synaptonemal complexes, at diplotene, reflects differences in their resistance towards degradation along the bivalent arms, regions of high probability for crossing over being less susceptible to breakdown. The segments which are preserved through early and mid diakinesis are assumed to have gained additional resistance as a consequence of crossing over in that region. 


\section{INTRODUCTION}

Chiasma formation and a regular disjunction of homologous chromosomes constitute the last events in the meiotic program. The ultimate success of meiosis, i.e., the formation of gametes containing balanced genomes, is thus dependent on a series of events occurring during diplotene anaphase I. These events include: chiasma formation, synaptonemal complex elimination, chiasma maintenance, bivalent contraction, congression of bivalents, co-orientation of homologous centromeres and disjunction of the homologues at anaphase I. These processes have been studied in the light microscope for about a century, and the general sequence of events as well as numerous species specific modifications have been described (see 3,12 for review).

In most organisms, however, only diakinesis nuclei are amenable to analysis while the chromatin of diplotene nuclei often is too diffuse to allow observations on bivalent conformation and chiasmata. Hence, comparatively little is known about this stage, and problems such as whether chiasmata are able to move - terminalize - during this stage are still under debate $(1,13,17$, 24). Furthermore, the analysis of the diakinesis metaphase I stages is generally performed on squashed cells or air-dried preparations, treatments which destroy the three dimensional organization of the nuclei.

The techniques of serial sectioning and three dimensional reconstruction have allowed that these processes can be reinvestigated, this time at the ultrastructural level. A number of investigations, in particular of the diplotene stage, have thus shown that in organisms where meiotic crossing over occurs synaptonemal complex segments are temporarily retained at the site of crossing over, and subsequently replaced by chromatin chiasmata, while the synaptonemal complex or a derivative thereof is present up to metaphase I in organisms where crossing over is absent (see 20 for review). It is thus apparent that the synaptonemal complex plays a major role in the regular disjunction of the homologues.

In contrast to the situation in nearly all other organisms, synaptonemal complex segments are retained between the homologues up to metaphase I in human spermatocytes $(19,23)$. The aim of the present study is to elucidate the rela- tionship between the synaptonemal complex segments and chiasmata by serial sectioning and three dimensional reconstruction of diplotene and diakinesis nuclei in order to see whether chiasma organization in the human male differs from that seen in other organisms. Furthermore, the number and distribution of chiasmata among and along the bivalents is compared with the number and distribution of recombination nodules and bars at pachytene $(10,18)$ and to chiasmata as seen in the light microscope at diakinesis $(11,15)$.

\section{MATERIALS AND METHODS}

\subsection{Materials}

The analysis was performed on biopsies from three normal fertile men undergoing vasectomy, the biopsies being the same as those used in previous studies of the zygotene and pachytene stages in human spermatogenesis $(6,9,18$, cases 3-5). Twelve nuclei covering the period from early diplotene to late diakinesis - prometaphase I were completely reconstructed, while five nuclei were serial sectioned and photographed but not reconstructed.

\subsection{Methods}

The procedures used for fixation, staining, electron microscopy and the subsequent reconstruction of the chromosome complement, as well as the methods for measuring the synaptonemal complex length, have been described previously $(6,9,18)$.

\section{RESULTS}

\subsection{The diplotene - prometaphase I period}

Spermatocytes at diplotene and diakinesis are rarely seen in the seminiferous tubules and light microscopical analysis of a large number of 2.5 $\mu \mathrm{m}$ plastic sections was required to locate clusters of cells in these stages. While cells belonging to the same cluster generally are at the same stage of development at other prophase stages, these clusters often contained cells ranging in development from early diplotene to prometaphase I, which, together with the scarcity of diplotene and diakinesis cells in general, shows that this interval is short compared to other stages of the prophase. The 17 analyzed cells fall into three groups - diplotene, diakinesis and diakinesis- 
prometaphase I transition - the cells in each group being characterized by typical common features. Nuclei allocated to diplotene and diakinesis were further divided into early, mid and late substages primarily based on the pattern of synaptonemal complex elimination. The changes in centriolar behaviour and nuclear morphology are described in the following section while detailed descriptions of centromere regions, synaptonemal complex elimination and $\mathrm{XY}$ bivalents are contained in sections 3.2 - 3.4.

\subsubsection{Early diplotene}

The onset of synaptonemal complex elimination from the fully paired pachytene bivalents marks the beginnning of the diplotene stage. The two analyzed early diplotene nuclei, viz. no. 56, 65 , contained 138 and $139 \mu \mathrm{m}$ of intact synaptonemal complex and numerous pieces of partially degraded lateral components and central regions. Reconstruction of the entire bivalent complement was possible in both nuclei by tracing the intact segments of synaptonemal complex as well as its remains from telomere to telomere. As at pachytene, the telomeres were still attached to the inner membrane of the nuclear envelope.

The total complement length of the nuclei amounted to 213 and $206 \mu \mathrm{m}$, which is about the same as the mean complement length of $198 \mu \mathrm{m}$ measured at late (stage 7) pachytene (9).

Additional characteristics of early diplotene cells are the following: (Figures 1 and 2): (A) The nuclei are large and spherical and the centrioles which have duplicated during pachytene (9) are located in the vicinity of each other, one of them positioned farther away from the nuclear envelope than the other. Both pairs are surrounded by a granular matrix from which a few microtubules emerge. (B) The chromatin of the autosomal bivalent arms is of uniform density except for a few regions of more compact chromatin, a pattern reminiscent of the chromatin organization at pachytene stage 7 where regions of uniformly dense chromatin alternate with compacted, irregular chromatin masses along the synaptonemal complex (9). (C) The fine structure of the $\mathrm{XY}$ bivalent is similar to that at late pachytene, the lateral components of the $\mathrm{X}$ and $\mathrm{Y}$ chromosomes being continuous over longer distances.
(D) The centromeric chromatin is in all bivalents differentiated into a sphere, more condensed than the chromatin of the bivalent arms, and a highly electron dense cup-like part which in most cases is located peripherally in the centromeric chromatin. (E) A gradual reduction in the number of nucleoli was observed during pachytene, the mean number at pachytene stage 7 amounting to 0.3 bivalent-associated and 0.6 free nucleoli per nucleus (9). In agreement with this, none of the diplotene and diakinesis nuclei contained nucleoli associated with acrocentric bivalents and elimination of the small, free nucleolus observed in some of the early diplotene nuclei was complete by late diakinesis. (F) As in pachytene stage 7 , the secondary constrictions on bivalents 1 and 9 can be identified, the former by a region of paracentromeric chromatin considerably larger than those of other bivalents whereas the secondary constriction on bivalent 9 exhibits the dimorphic structure typical of pachytene nuclei. Both chromatin differentiations remain unchanged throughout diplotene and diakinesis. The subterminal marker previously described (2) on the short arm of bivalent 1 is recognizable at all stages as a sphere of coarse fibers containing one or two electron dense bodies of which one is associated with the chromatin.

\subsubsection{Mid diplotene}

Five nuclei, viz. no. 51, 53, 54, 74, 77, were assigned to the mid diplotene stage and in three of these, viz. no. 51, 53, 54, reconstruction of the entire bivalent complement was possible by tracing intact segments of synaptonemal complex, remnants of the complex and the chromatin contour (Figures 3 and 4).

The mean synaptonemal complex length amounted to $74 \mu \mathrm{m}$ while the length of the bivalent complement was $206 \mu \mathrm{m}$, i.e. about the same as at late pachytene and early diplotene. The early elimination of synaptonemal complexes is thus not accompanied by a reduction of the bivalent lengths. The nuclei contained two morphologically different types of synaptonemal complex segments, one being similar to the synaptonemal complexes at pachytene while the other appeared partially degraded (Figure 4). Most of the telomeres were attached to the nu- 


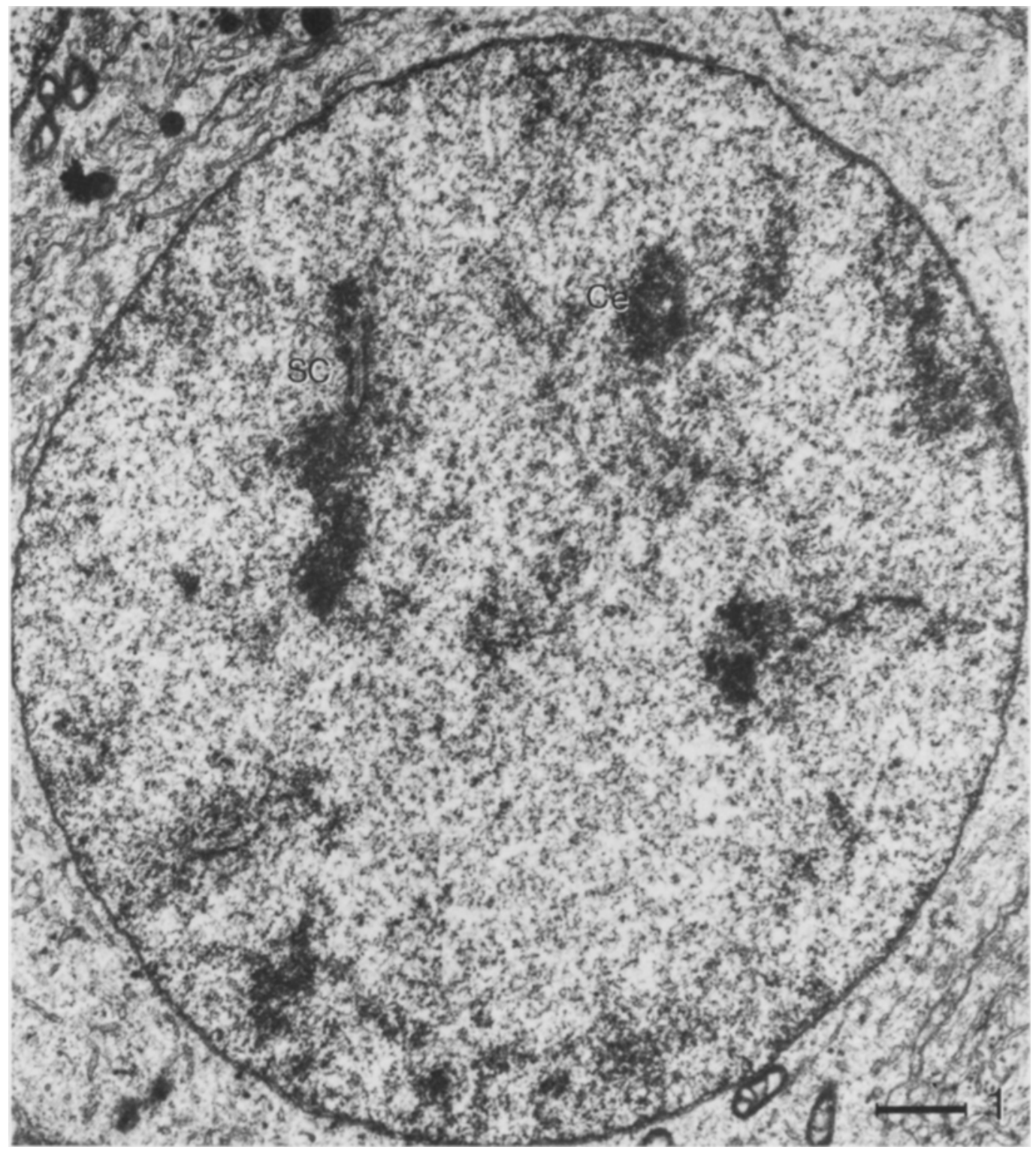

Figure 1. Survey micrograph of an early diplotene nucleus. Ce. Centromere region. SC, synaptonemal complex. $(\mathrm{Bar}=1 \mu \mathrm{m})$

clear envelope and often contained remnants of lateral components.

The size and shape of the nucleus as well as the morphology of the centrioles (Figure 5) are as at early diplotene. The chromatin of the bivalent arms is uniformly dense along the synaptonemal complex although slightly more compacted than at early diplotene. Unpaired lateral components or their remnants were not observed in the XY bivalent and the appearance of its chromatin resembled that of the autosomes.

\subsubsection{Late diplotene}

Late diplotene cells are very similar to cells at 


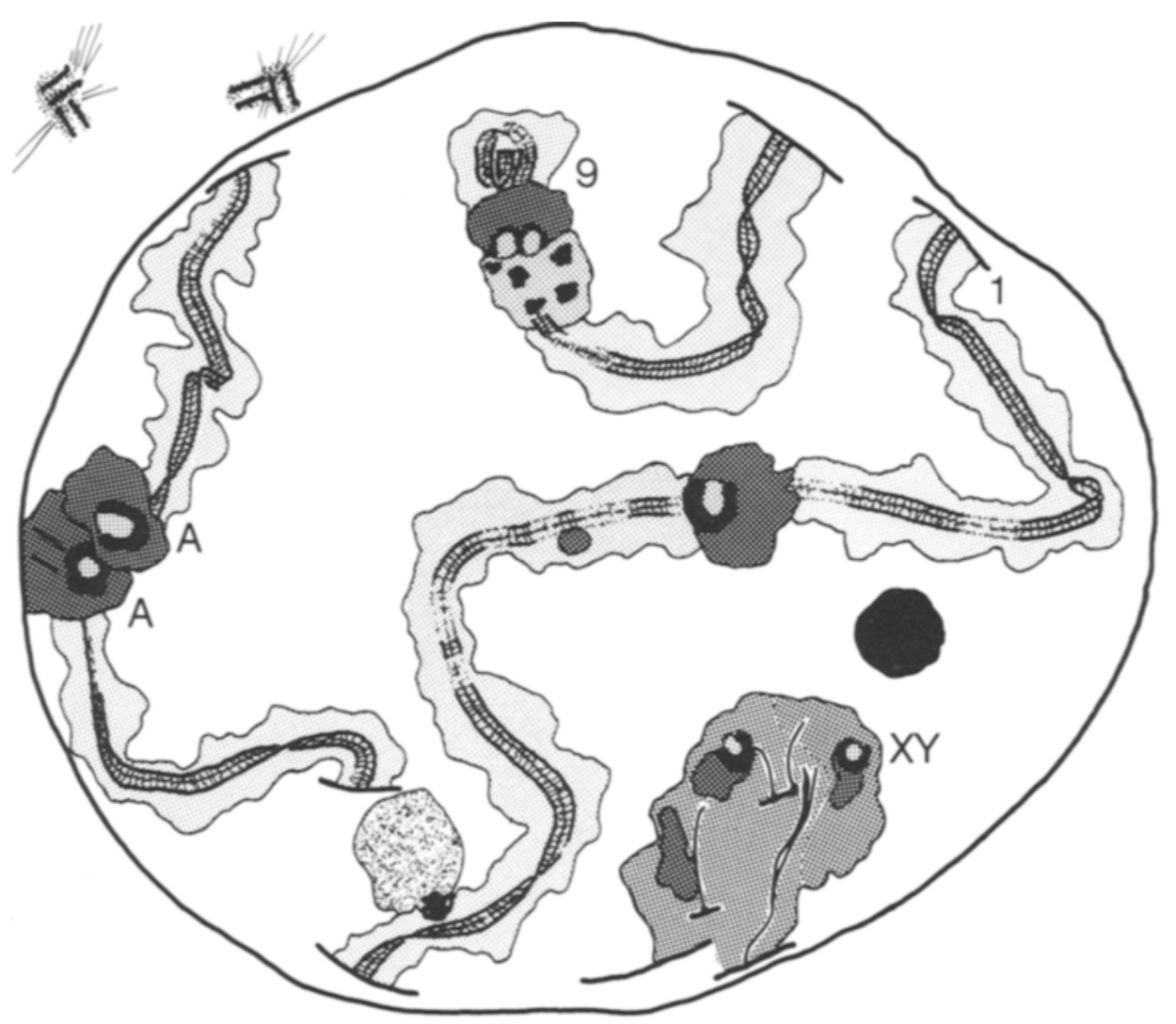

Figure 2. Partial reconstruction of an early diplotene nucleus (number 56 ) showing bivalents 1, 9, the $\mathrm{XY}$ bivalent and two acrocentric bivalents (A). The condensed chromatin of the centromere regions are the dark hatched areas associated with the black cup surrounding the lightly hatched prokinetochores. Bivalent 1 is recognized by marker 1, the granular area with a black patch near the telomere of the short arm. Bivalent 9 contains adjacent to its centromere region, the dimorphic structure identifying the secondary constriction. The free nucleolus is shown in black. The centromere regions, the nucleolus, the marker on bivalent 1 and the dimorphic structure on bivalent 9 are shown with the same hatching in the reconstructions in Figures 4, 7, 9,11 and 14.

mid diplotene and separate survey micrographs and reconstructions are not shown. Two of the four nuclei, viz. no. $30,40,52,55$, allocated to this stage were reconstructed, viz. no. 30,40 . Only 20 of the 22 bivalents could be traced in nucleus 30 as the chromatin of two bivalents was confluent with the XY bivalent.

The mean total synaptonemal length was 63 $\mu \mathrm{m}$ while the mean length of the bivalent complement length amounted to $155 \mu \mathrm{m}$, i.e. 78 percent of the pachytene length. Shortening of the bivalents has thus commenced at this stage. The two pairs of centrioles are several micrometers apart, the granular matrix surrounding the centrioles is prominent and microtubules extend over longer distances than at earlier stages.

\subsubsection{Early diakinesis}

The early diakinesis stage is represented by one nucleus, viz. no. 60, (Figures 6 and 7). Reconstruction of the individual bivalents at this and later diakinesis stages was performed by tracing the remains of the synaptonemal complex and the chromatin contour. The total length 


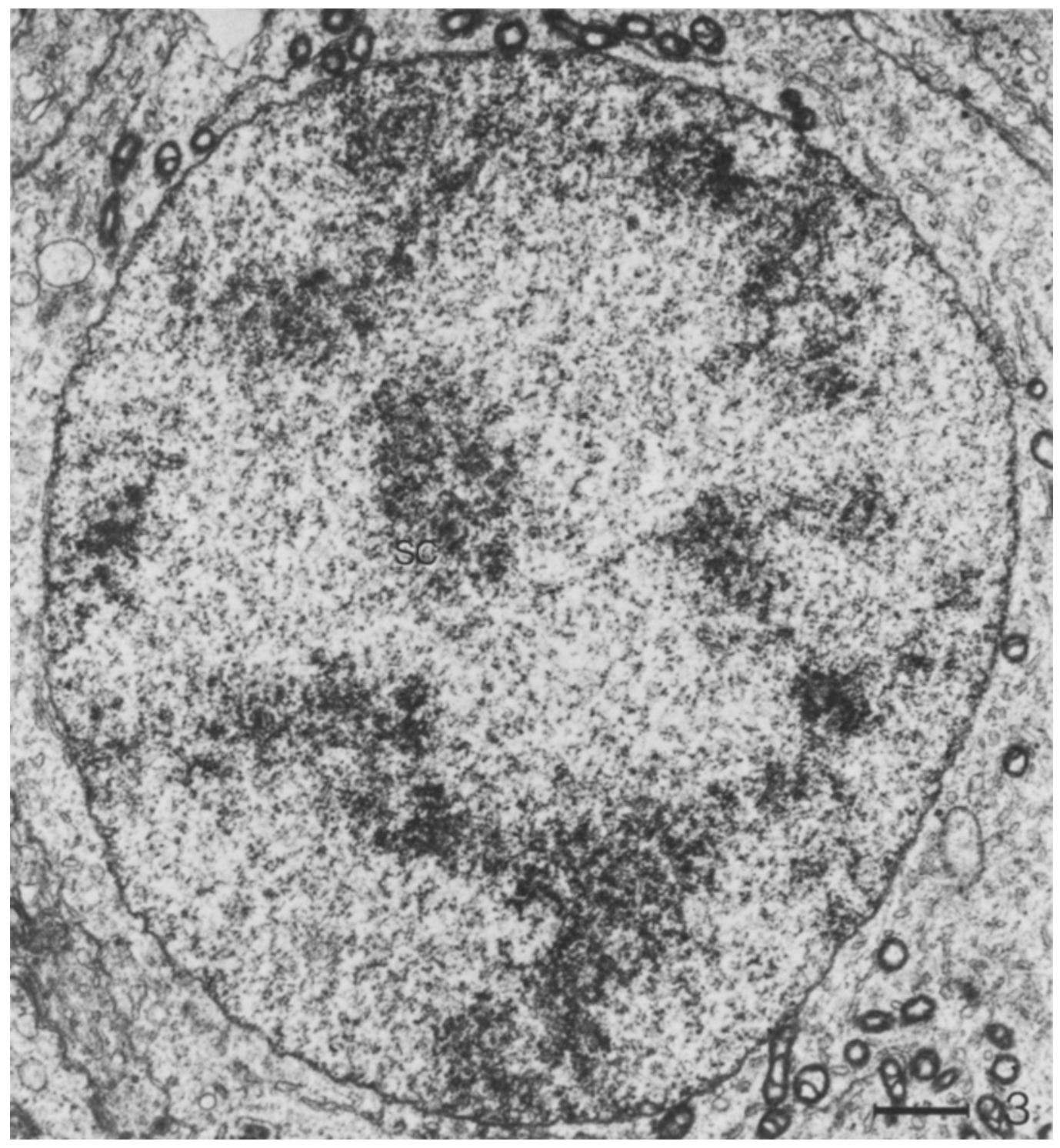

Figure 3. Survey micrograph of a mid diplotene nucleus. $\mathbf{S C}$, synaptonemal complex $($ Bar $=1 \mu \mathrm{m})$

of the synaptonemal complex segments amounted to $28 \mu \mathrm{m}$ whereas the total length of the bivalent complement and the length of individual bivalents could not be determined. The two homologues of each bivalent were in all cases aligned throughout their length, the contact region between the nonsister chromatids in most cases having a low chromatin density. The two pairs of centrioles are further apart than at late diplotene, associated with a larger number of $\mathrm{mi}$ crotubules and are located at the flattened side of the kidney-shaped nucleus.

\subsubsection{Mid diakinesis}

Two reconstructed nuclei, viz. no. 29, 50, were allocated to the mid diakinesis stage. As seen in the survey micrograph and the reconstruction (Figures 8 and 9) the nuclei are now heart-shaped 


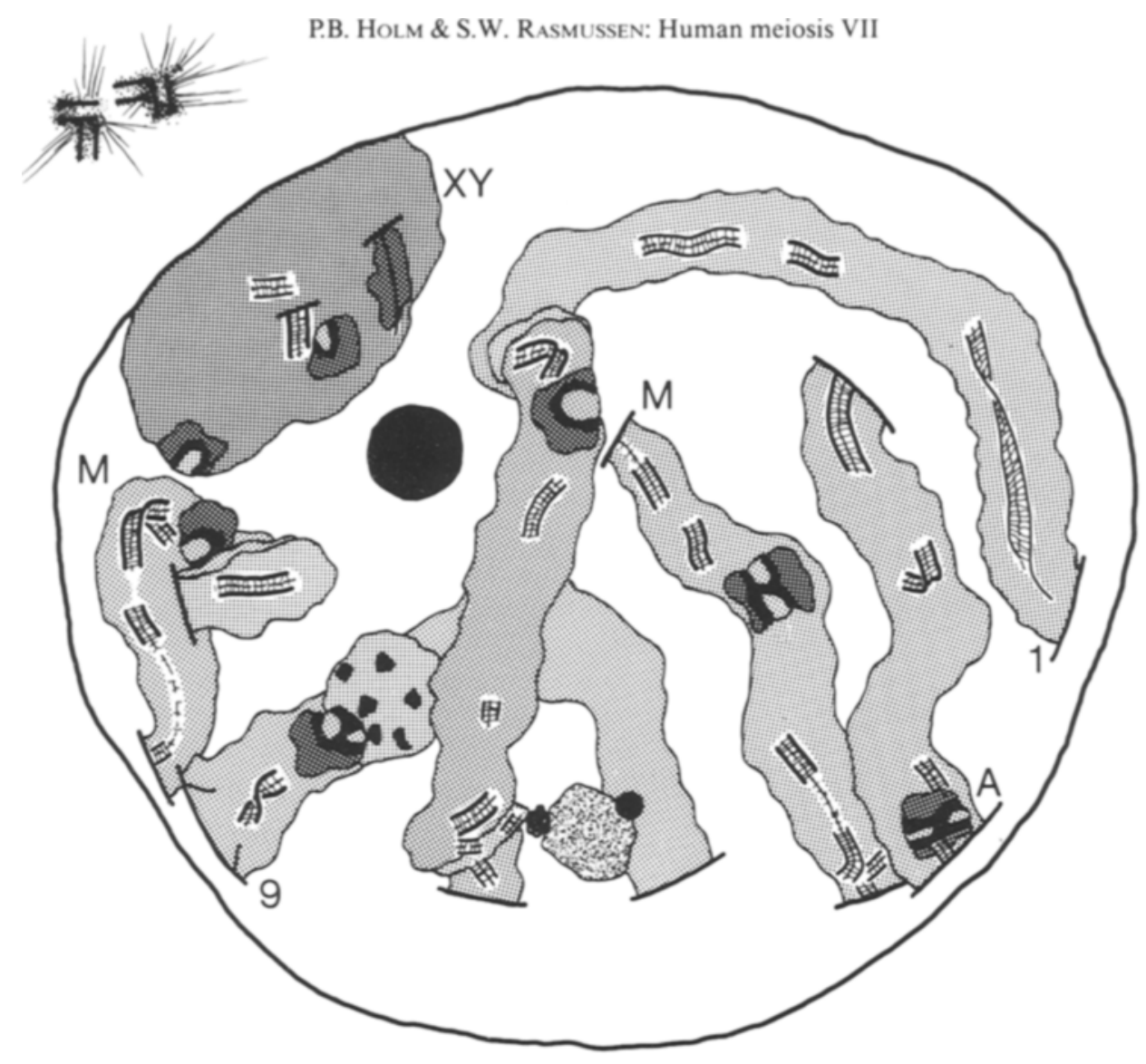

Figure 4. Partial reconstruction of a mid diplotene nucleus showing bivalents 1,9 , the XY bivalent, an acrocentric bivalent (A) and two meta-submetacentric bivalents (M). Lateral components of distinct synaptonemal complexes are shown with a thicker line than those of degrading complexes. See also the legend to Figure 2.

and one of the two centriole pairs is located in the indentation of the nuclear envelope. The bivalents are shorter and the chromatin is more condensed than at earlier stages but still contains distinct synaptonemal complex segments, their mean total length amounting to $32 \mu \mathrm{m}$.

\subsubsection{Late diakinesis}

At late diakinesis, the nucleus, viz. no. 67 , is cup-shaped (Figures 10 and 11) and both centriole pairs are located within the invagination. As shown in Figure 12, the centriole pairs are

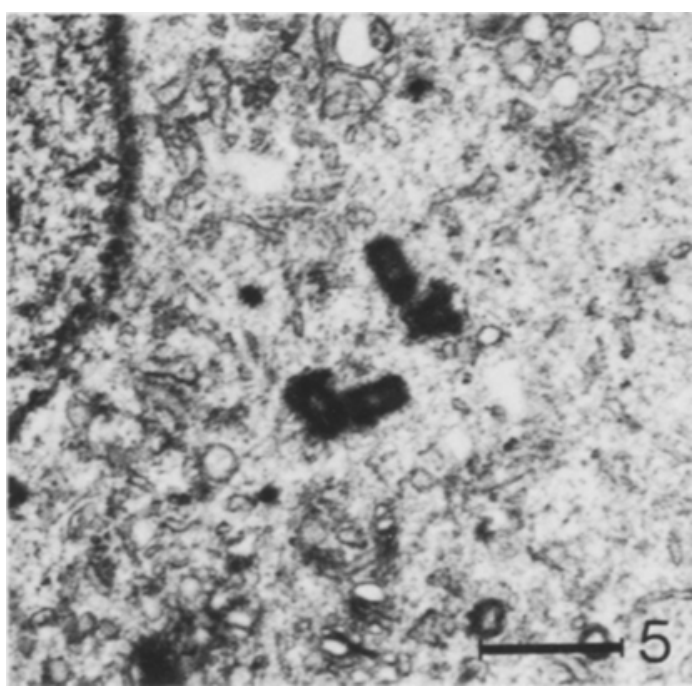

Figure 5. Two centriole pairs at mid diplotene. $(\operatorname{Bar}=1$ $\mu \mathrm{m})$ 


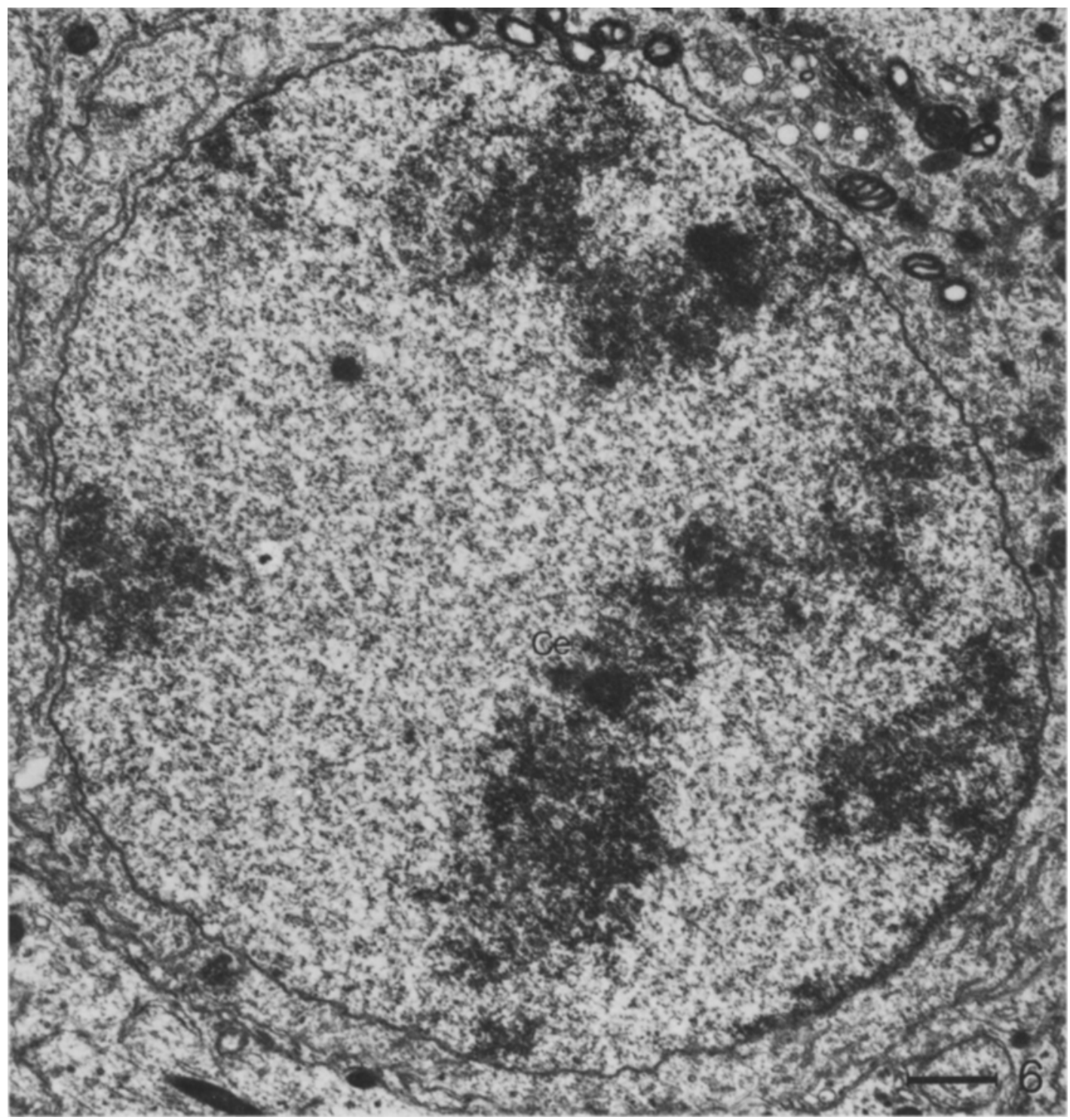

Figure 6. Survey micrograph of an early diakinesis nucleus. Ce, centromere region. $($ Bar $=1 \mu \mathrm{m})$

associated with a large amount of granular material from which numerous microtubules emerge. Nearly all microtubules are oriented parallel to the nuclear envelope or radiate out into the cytoplasm. The bivalents are shorter and more contracted than at mid diakinesis and most of the synaptonemal complex segments have fused into aggregates of varying shape. The 22 autosomal bivalents were traced by their chromatin contour and in most cases it was possible also to distinguish the two homologues, the narrow space between them containing less compacted chromatin. Partial separation of homologous centromere regions, telomere and interstitial regions was only rarely observed (Figure 11).

\subsubsection{The diakinesis - prometaphase I transition}

Two nuclei, viz. no. 32,64 , of which one was 


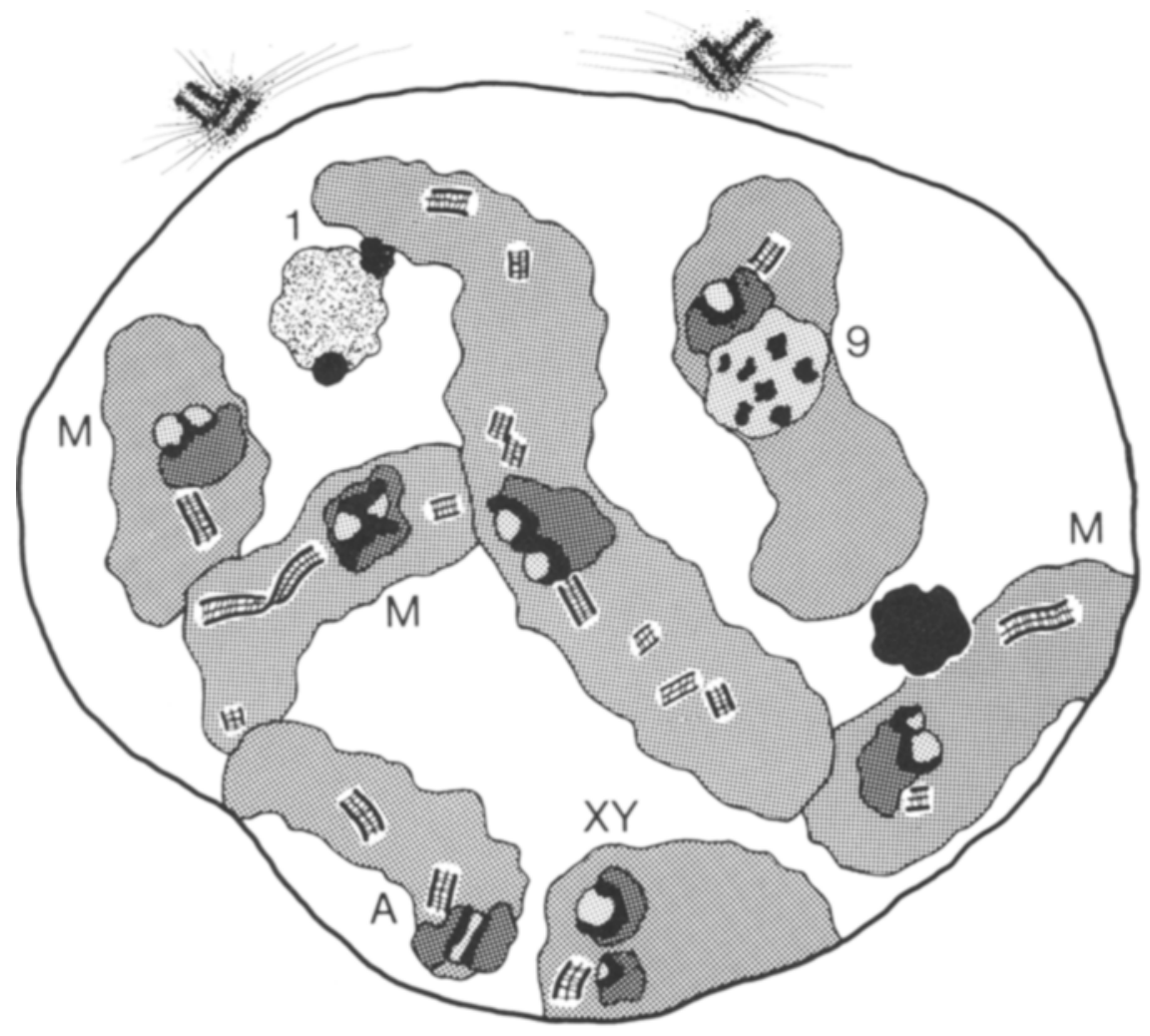

Figure 7. Partial reconstruction of an early diakinesis nucleus showing bivalents 1,9 , the XY bivalent, an acrocentric bivalent (A) and three meta-submetacentric bivalents (M). Lateral components of distinct synaptonemal complexes are shown with a thicker line than those of indistinct synaptonemal complexes. See also the legend to Figure 2.

completely serially sectioned, represent the transition between late diakinesis and prometaphase I. The period is characterized by breakdown of the nuclear envelope into vesicles and sheets of membranes. It appears from Figures 13 and 14 that the remaining continuous portion of the nuclear membrane still envelops the bivalents confining them to a smaller volume than at earlier stages. (The volume of nuclei from early diplotene to late diakinesis is approximately 800 $\mu \mathrm{m}^{3}$ while that of nucleus 32 is only $300 \mu \mathrm{m}^{3}$ ). The two centriole pairs were located at opposite poles in the partially sectioned nucleus. (Only one centriole pair was included in the complete series through nucleus 32 ). In both nuclei, the bivalents were heavily condensed, several bivalents forming large confluent chromatin masses and it was only in few cases possible to delimit individual bivalents with certainty.

\subsection{The centromere region and the kinetochore}

The centromere region consists at early pachytene of a single region of uniformly condensed chromatin. The size and density of this region increase during pachytene and by late pachytene (stages 5-7,9) a single sphere of medium dense material - the prokinetochore - develops inside the large condensed region associated with the synaptonemal complex. The prokinetochores become surrounded by a "cup" of highly electron dense material at early diplotene (Figures 1, 2 and 15 a and $b$ ) and from early di- 


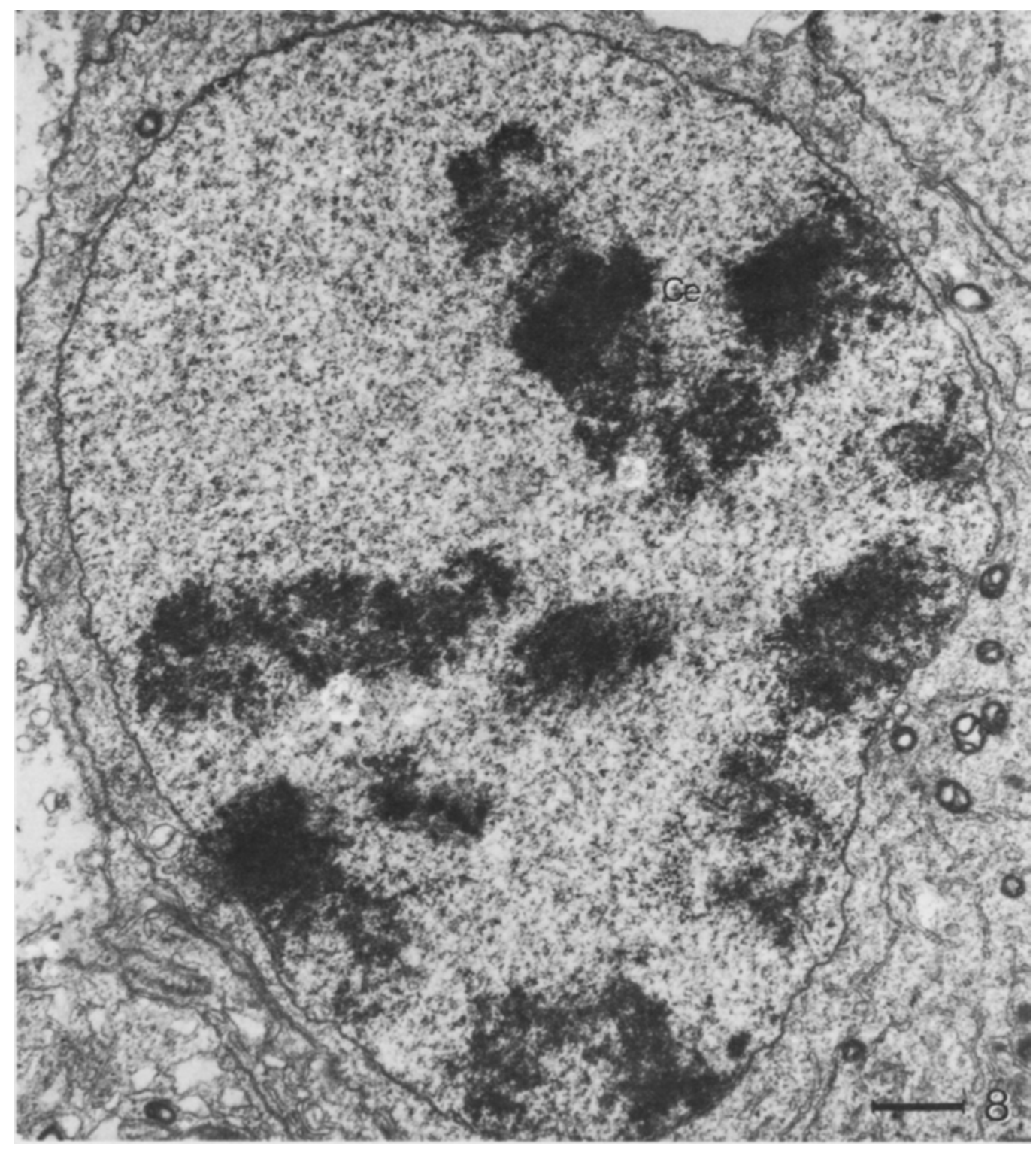

Figure 8. Survey micrograph of a mid diakinesis nucleus. Ce, centromere region. $($ Bar $=1 \mu \mathrm{m})$

plotene to mid diakinesis all bivalents contain a single centromere region with, in most cases, two confluent cups each with a prokinetochore. In rare cases, the two cups and prokinetochores are located in different parts of the condensed chromatin of the centromere region while complete separation of the two homologous centromere regions was not observed between early di- plotene and mid diakinesis. The two electron dense, often still confluent, cups open during late diplotene and early diakinesis and expose both prokinetochores on the surface of the centromere region (Figures 4, 6, 7 and $15 \mathrm{c}$ ). This development continues into mid diakinesis where the two prokinetochores are often completely exposed facing each other or lying side by 


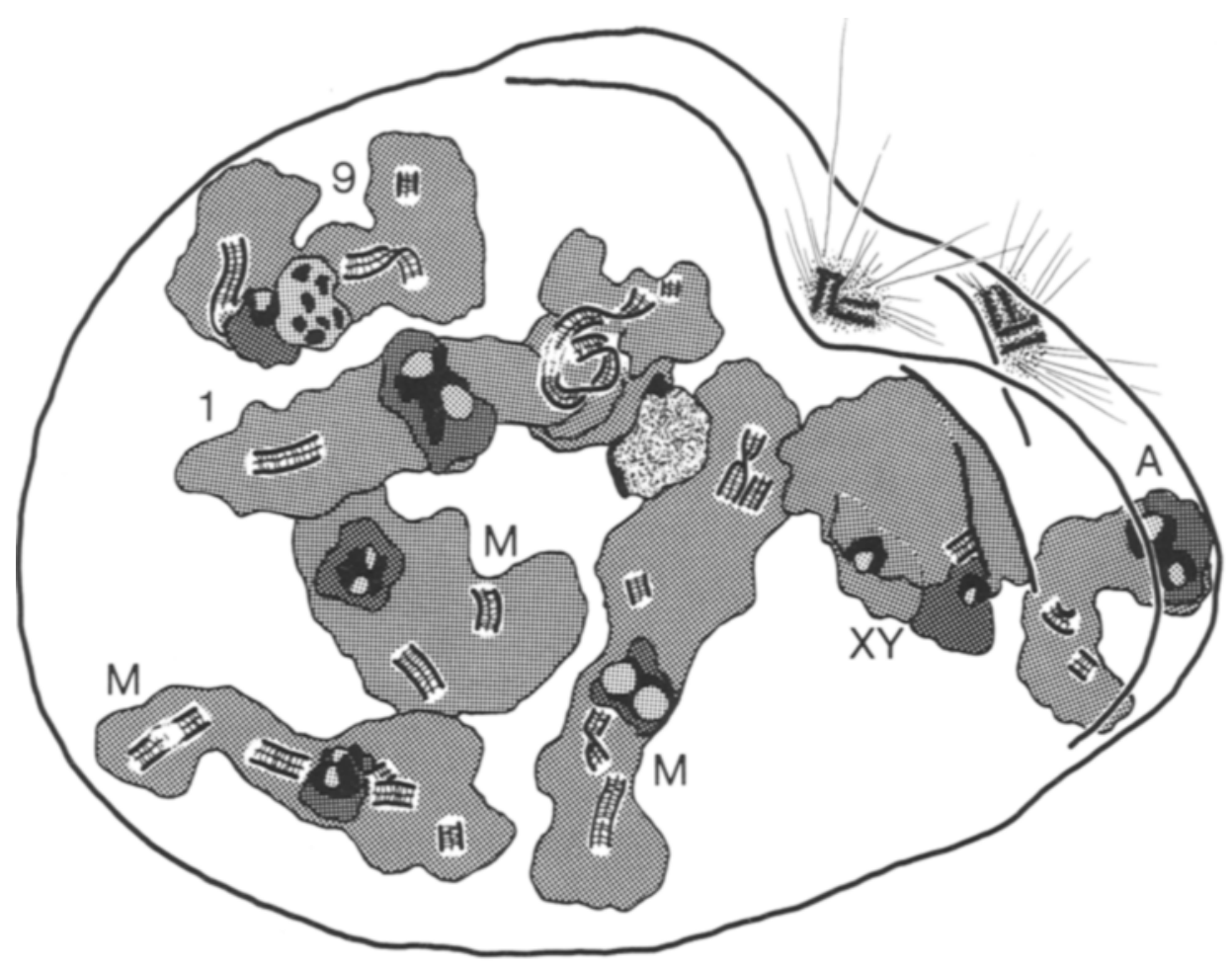

Figure 9. Partial reconstruction of a mid diakinesis nucleus showing bivalents 1,9 , the XY bivalent, an acrocentric bivalent (A) and three meta-submetacentric bivalents (M). Lateral components of distinct synaptonemal complexes are shown with a thicker line than those of indistinct synaptonemal complexes. See also the legend to Figure 2.

side (Figures 8 and 9). Favorable sections at this stage sometimes reveal a faint line within the prokinetochore material, possibly the early kinetochore plate (Figure 15 d). Most prokinetochores, however, appear to lack recognizable indications of a kinetochore plate either due to intranuclear asynchrony or because of unfavourable angles of section.

By late diakinesis, the dense cups of all bivalents are stretched into flattened plates to which the prokinetochore material is associated (Figures 11 and $15 \mathrm{e}$ ) and in a few cases the kinetochore plate can be identified as a faint line. Partial individualization of homologous centromere regions were observed in some bivalents but only in three cases were the homologous cen- tromere regions, but not the remainder of the homologues, completely separated.

The final organization of the kinetochores is attained at the diakinesis-promataphase transition, the two homologous kinetochores of the bivalent consisting of two crescent-shaped discs (Figures 13,14 and 15 f) covered on both sides by material of medium electron density. In the completely sectioned late diakinesis nucleus, the expected 23 pairs of kinetochores could not, however, be identified possibly in part due to asynchronous development of the bivalents or more likely as a result of the difficulties in identifying kinetochores in sections parallel to the disc. In those bivalents where kinetochores could be identified, they were always double and were as- 


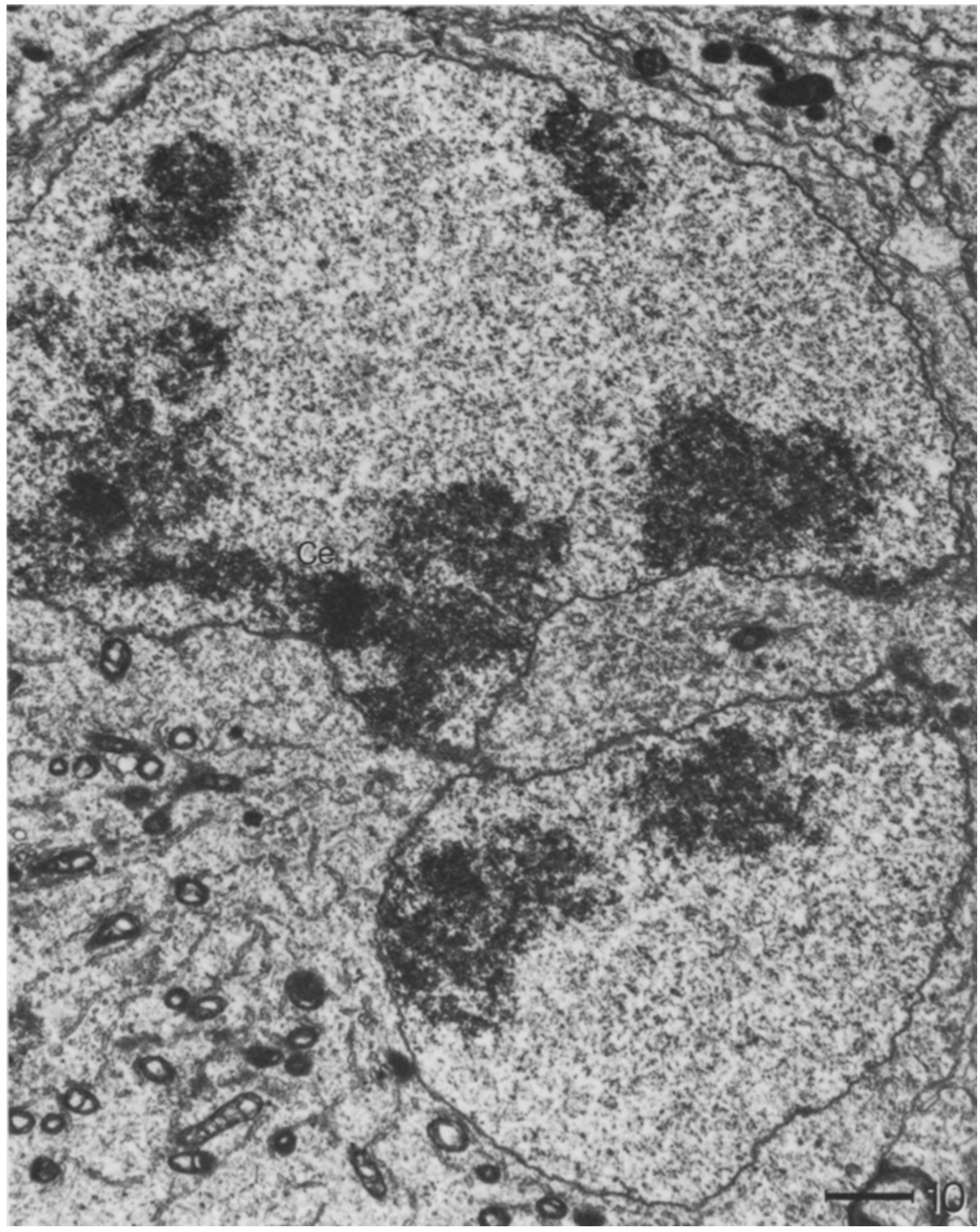

Figure 10. Survey micrograph of a late diakinesis nucleus. Ce, centromere region. (Bar $=1 \mu \mathrm{m})$

sociated with a single, common region of uniformly condensed chromatin of a higher electron density than that of the remainder of the bivalents.

\subsection{Elimination of the synaptonemal complex} 3.3.1. Morphological aspects

As illustrated by the reconstruction in Figure 2 , degradation of the constituents of the synap- 


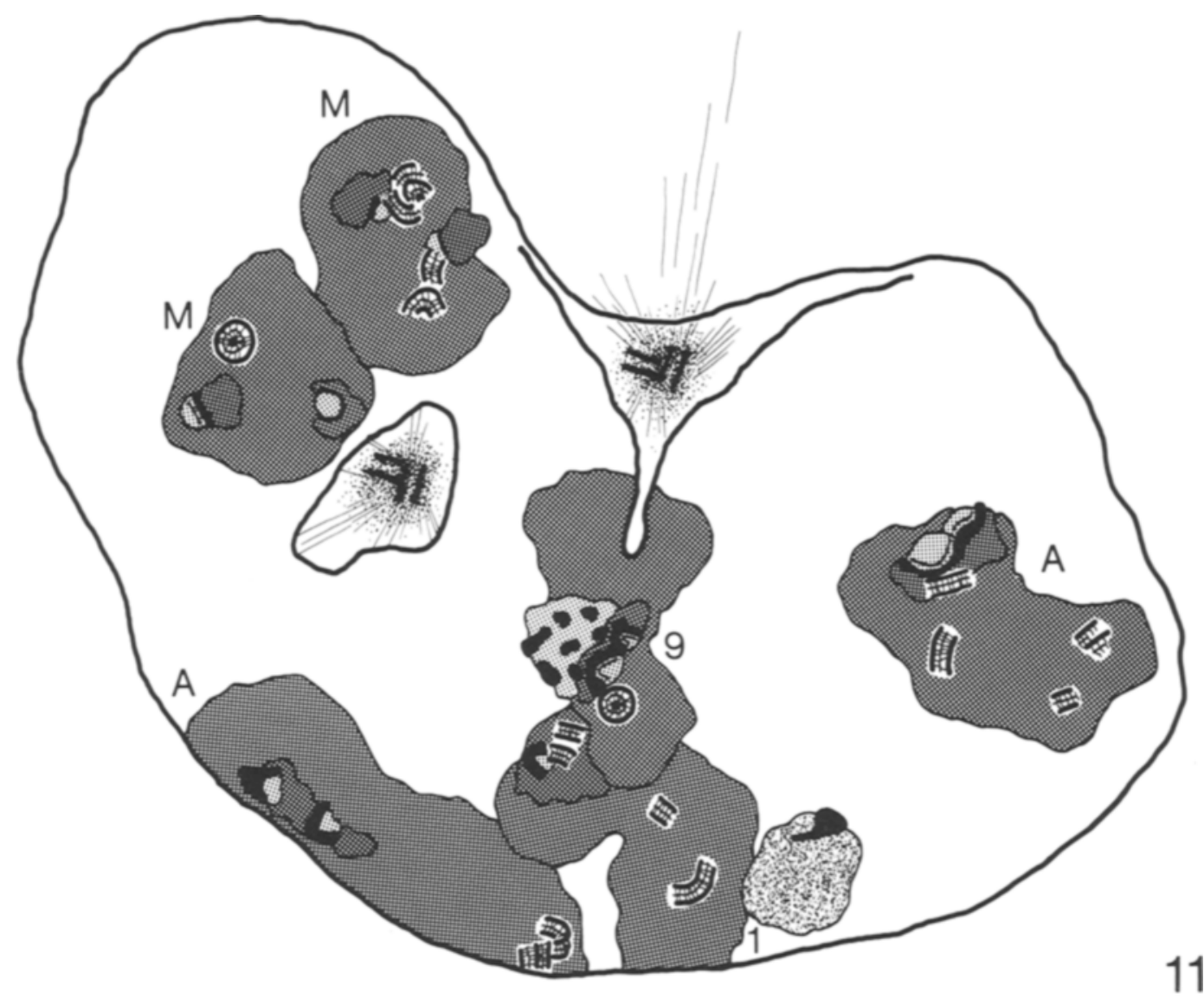

Figure 11. Partial reconstruction of a late diakinesis nucleus showing bivalents 1,9 , two acrocentric bivalents (A) and two meta-submetacentric bivalents (M). See also the legend to Figure 2.

tonemal complex was in progress in the two early diplotene nuclei, segments of intact synaptonemal complex alternating with regions either devoid of complex or with more or less intact remnants of lateral components and central regions (Figure 16). At this, as well as in the succeeding stages, intact segments of synaptonemal complex were never found inside or in the immediate vicinity of the centromere region.

The reduction in length of the synaptonemal complex segments observed at mid-late diplotene is accompanied by a structural differentiation of the remaining segments into two morphologically different types. Most of the seg-

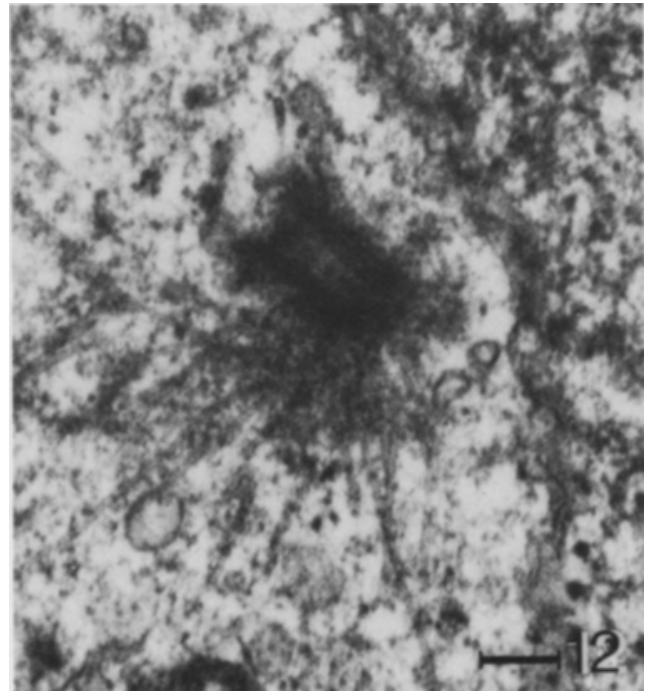

Figure 12. Micrograph of a centriole at late diakinesis. $(\mathrm{Bar}=0.2 \mu \mathrm{m})$ 

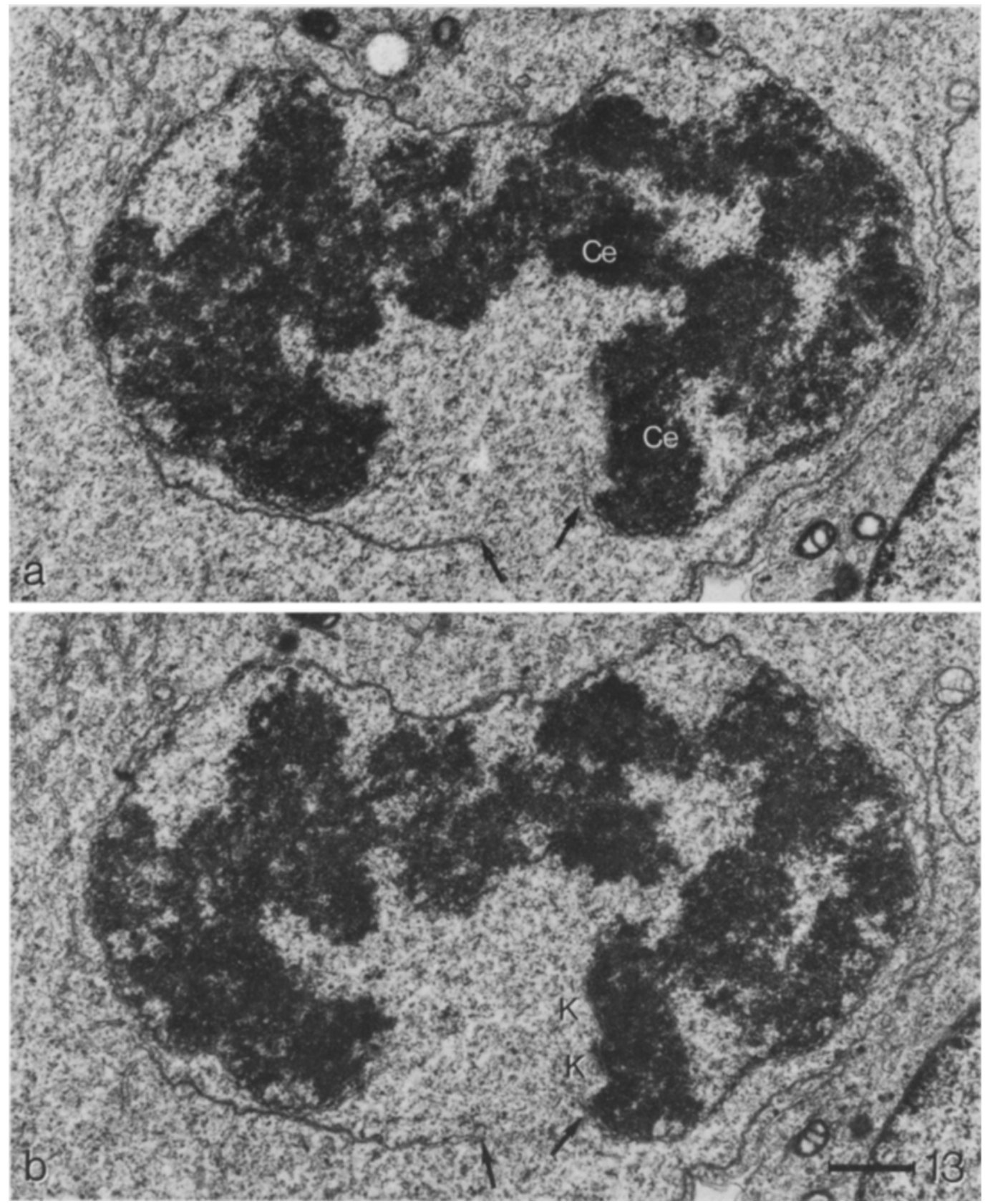

Figure 13. Two consecutive sections through a late diakinesis-prometaphase I nucleus. The breakdown of the nuclear envelope has commenced, a discontinuity being indicated by the arrows. Ce, centromere region. $\mathrm{K}$, kinetochore. $(\mathrm{Bar}=1 \mu \mathrm{m})$

ments appear structurally identical to synaptonemal complexes at pachytene or, at late diplotene, with even thicker and denser lateral components. The remaining segments were still tripartite but with inconspicuous lateral components and central regions, indicating partial degradation (Figure 4 ). This was most readily observed in two of the reconstructed mid di- 


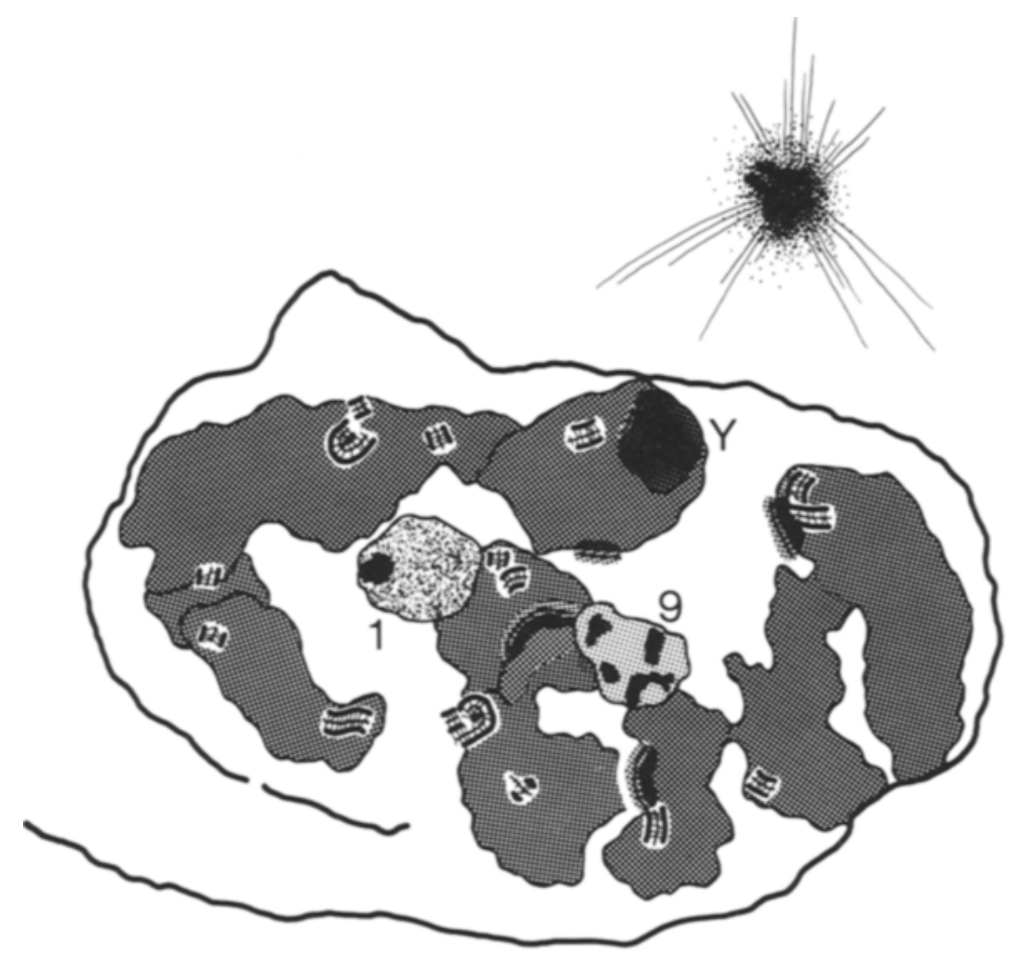

Figure 14. Partial reconstruction of a late diakinesis-prometaphase I nucleus. Note that the discontinuous nuclear membranes still envelop all bivalents. The chromatin of the bivalents is confluent and it is not possible to delimit the individual bivalents. The $\mathrm{XY}$ bivalent with the condensed long arm of the $Y$ chromosome is shown as well as the marker on bivalent 1 and the dimorphic structure characteristic of the secondary constriction on bivalent 9 . Most synaptonemal complex fragments are disorganized and located at the periphery of the bivalents.

plotene,viz. no. 53,54, and the late diplotene nuclei, while the difference between the two types was less prominent in the third mid diplotene nucleus, viz. no. 51. As shown in Figure 17 , segments of the distinct type end abruptly without partially degraded flanking regions. Small spheres of chromatin-like material were associated with the middle part or the ends of most distinct synaptonemal complex segments during diplotene. It is conceivable that these structures represent chromatin chiasmata but as morphologically similar condensations are present also in regions outside the synaptonemal complex further conclusions on the nature of these chromatin differentiations are not possible.

The homologues remain combined by well-defined and distinct synaptonemal complex segments during early and mid diakinesis (Figures 18 and 19), the segments being somewhat shorter than at mid-late diplotene. Some segments, however, appear slightly disorganized, and by late diakinesis most segments no longer combine the homologues (Figure 20) but are present as small aggregates of short fragments or form semicircular to circular structures which, in a few cases, have fused into larger aggregates or cylindrical polycomplexes (Figures 11, 12 and 21). In several cases, a resumptive chromatin chiasma, 

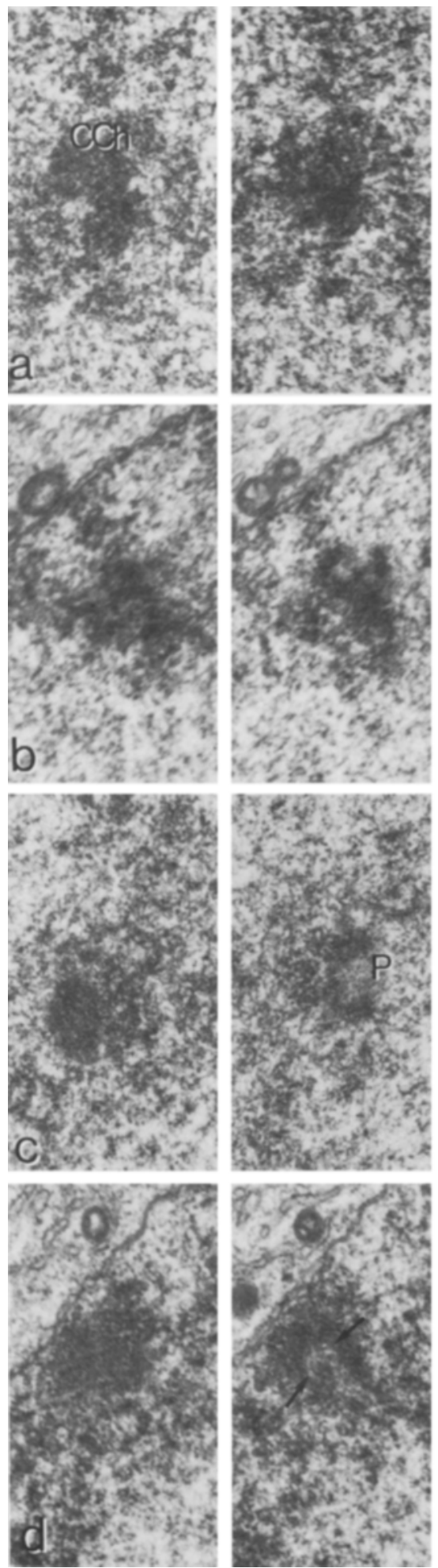
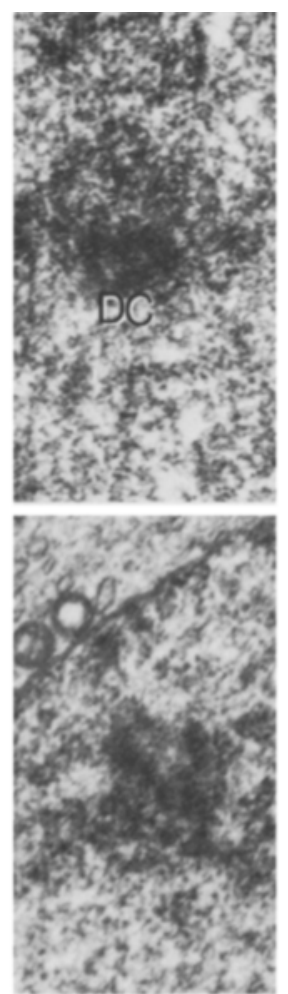
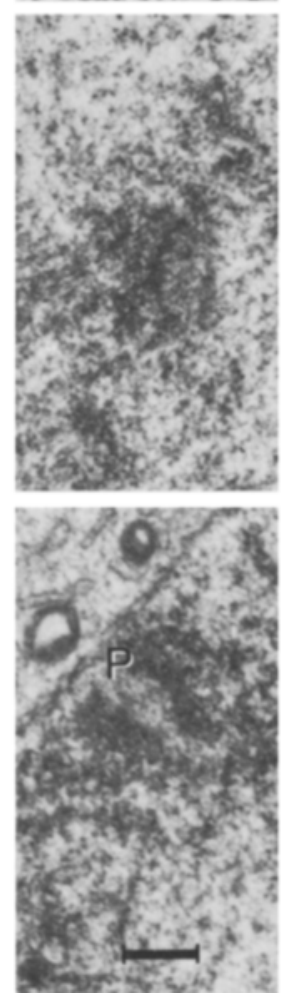
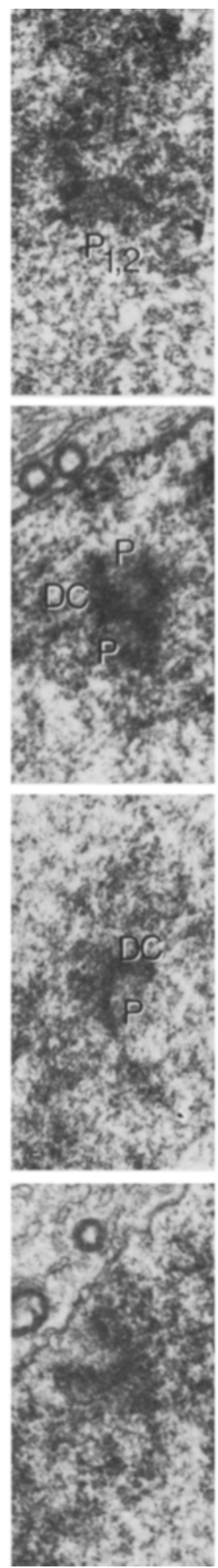
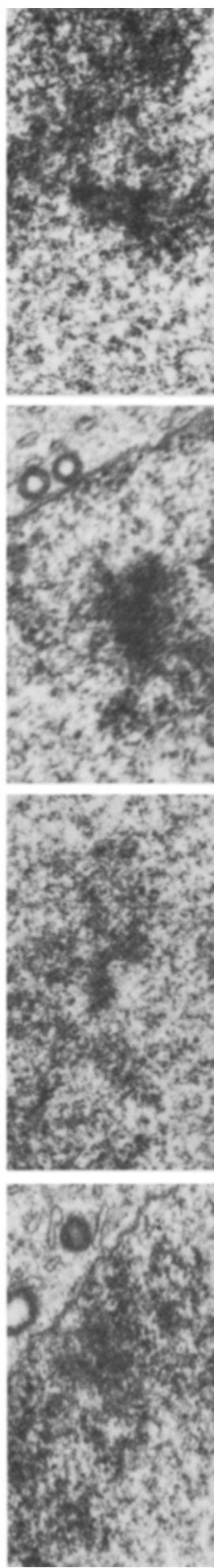

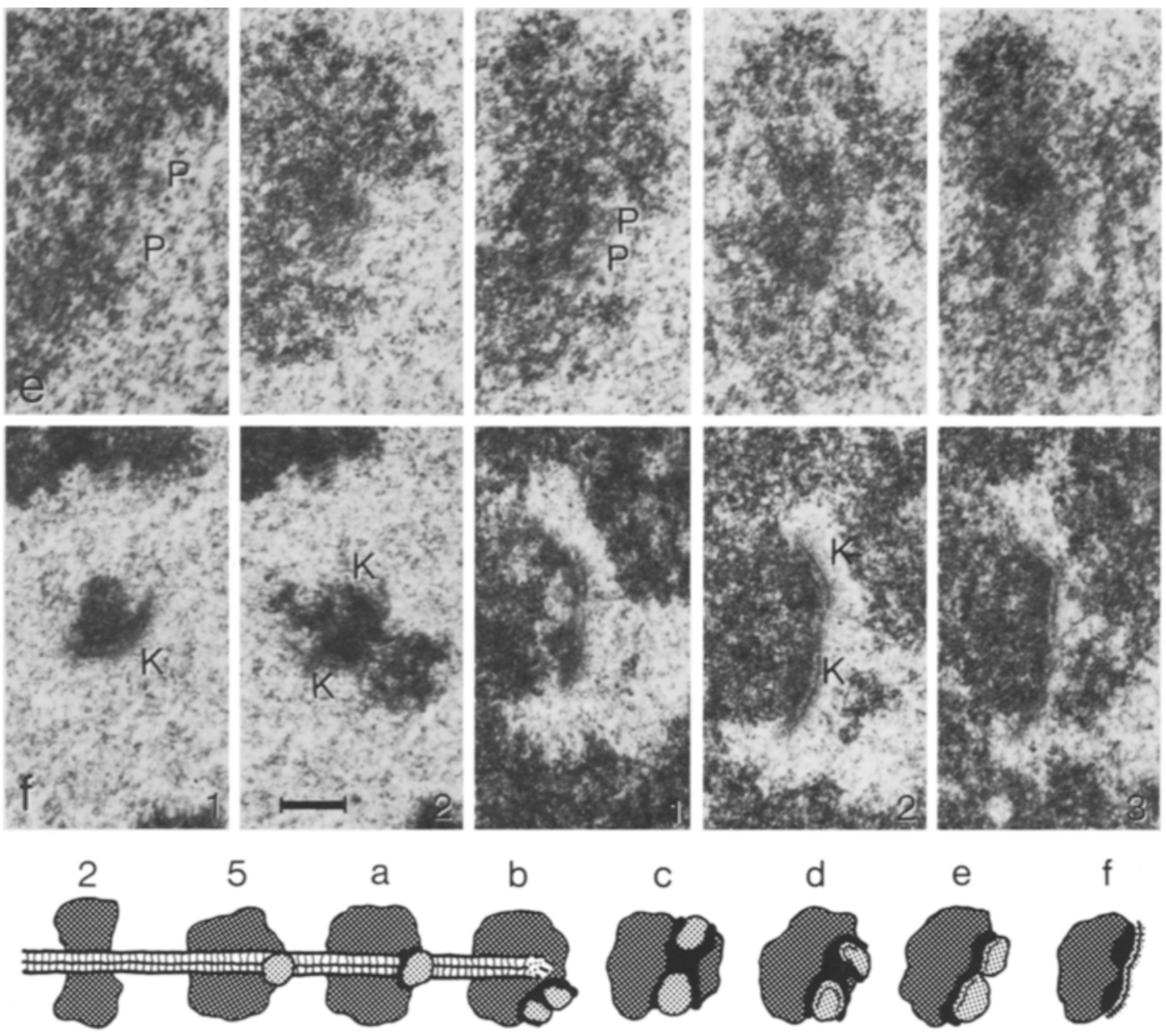

g

Figure 15. The development of the kinetochore from early diplotene to late diakinesis illustrated by a series of consecutive sections at (a) early diplotene, (b) mid diplotene, (c) late diplotene, (d) mid diakinesis, (e) late diakinesis and (f) late diakinesis-prometaphase $\mathrm{I}$. The schematic drawing in Figure ( $\mathrm{g}$ ) includes, in addition to the above stages, pachytene stages 2 and 5. P, prokinetochore. DC, dense cup containing the prokinetochore. K, kinetochore. $\mathrm{CCh}$, centromeric chromatin. The arrows in Figure d denote the early kinetochores. $(\mathrm{Bar}=0.5 \mu \mathrm{m})$

consisting of a chromatin bridge between the homologues was identified in the vicinity of partly disorganized synaptonemal complex (Figure 22).

At the late diakinesis-prometaphase I transition, the synaptonemal complex segments no longer appear to combine the homologues. The majority of the synaptonemal complexes have fused into small polycomplexes at the periphery of the bivalents and the elimination of the synap- tonemal complex from the bivalents is thus about to be completed. As described previously (19), these polycomplexes remain in contact with the surface of the bivalents, often near the centromere region until, by metaphase I-anaphase I, they are released into the cytoplasm.

\subsubsection{Quantitative aspects of synaptonemal complex elimination}

It can be seen from Table I that the total length 

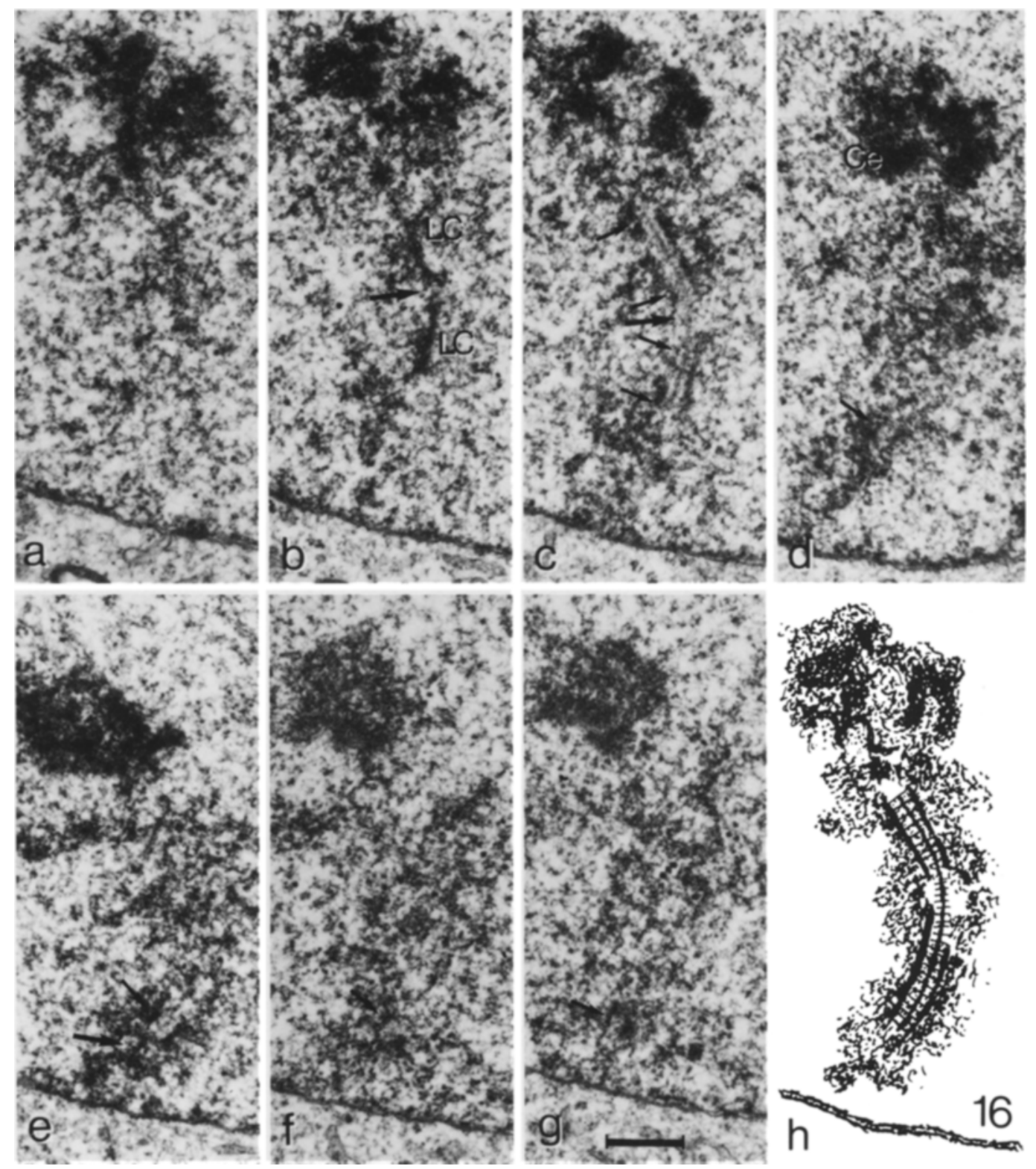

Figure 16. Seven consecutive longitudinal sections through a bivalent arm at early diplotene. The series includes most of the arm and reveals in Figures $b$ and $c$ two sites where breakdown of the synaptonemal complex has just commenced (denoted by arrows). As shown by the reconstruction in Figure h, one of the initiation sites for the degradation is near the centromere region and the second approximately in the middle of the arm. A third site is present at the telomere region (not included in this sequence). The degradation of the lateral components seems to precede the disintegration of the central region of the complex, the middle region of the complex segment lacking both lateral components but having an apparently intact central region. The intact stretches of complex are ultrastructurally very similar to those of late pachytene nuclei and appear to be associated with the chromatin of both homologues along the entire length of the lateral components. Note that the synaptonemal complex segments are devoid of recombination nodules and bars. A chromatin bridge, denoted by an arrow in Figure e combines the two lateral components of the synaptonemal complex segment. Ce, centromere region. LC, lateral component. The synaptonemal complex segments are denoted by small arrows. $(\mathrm{Bar}=0.5 \mu \mathrm{m})$ 
of all synaptonemal complex segments decreases during diplotene until about $30 \mu \mathrm{m}$ of complex is left at early diakinesis. Thereafter, the length remains constant. The number of distinct synaptonemal complex segments on the other hand remains almost constant during diplotene (mean number of 72) and is nearly equal to the total number of segments at early-mid diakinesis (mean number of 62). As shown in Figure 23, segments at early-mid diakinesis are rather uniform in length, the majority being shorter than 1 $\mu \mathrm{m}$ whereas distinct fragments at mid-late diplotene are more heterogeneous in size.

These observations allow the following conclusions: Degradation of the synaptonemal complex is initiated at a low number of sites producing about 70 synaptonemal complex segments of typical pachytene morphology. At mid and late diplotene the length of these segments is reduced by the release of fragments from the ends of the distinct stretches, fragments which due to further degradation appear structurally indistinct. Eventually both the lateral components and the central regions of these fragments are broken down into subunits too small to be recognized.
During diakinesis no further reduction in synaptonemal complex length occurs, but some segments are partially degraded. The resultant indistinct and the remaining distinct segments are finally released from the homologues and reassemble into synaptonemal polycomplexes at late diakinesis-prometaphase I.

\subsection{The XY bivalent}

\subsubsection{Diplotene}

The morphology of the XY bivalent at early diplotene is very similar to that at pachytene stage 7 (Figures 2 and 24). The lateral components are nearly continuous and it is usually possible to trace most of the $Y$ chromosome. As at pachytene stage 7 , the chromatin of its long arm contains local condensations.

At mid-late diplotene, the chromatin morphology of the XY bivalent approaches that of the autosome: most lateral components have disappeared and reconstruction of the $X$ and $Y$ chromosomes is no longer possible (Figures 4 and 25). The position of the $Y$ chromosome can be established by its centromere which is always located in the vicinity of the nuclear envelope

Table I.

Mean number and length per nucleus of distinct and indistinct synaptonemal complex segments (SCS) at diplotene and diakinesis.

\begin{tabular}{|c|c|c|c|c|c|c|c|}
\hline \multirow[t]{2}{*}{ Stage } & \multirow{2}{*}{$\begin{array}{l}\text { Nucleus } \\
\text { number }\end{array}$} & \multicolumn{3}{|c|}{ SCS length $(\mu \mathrm{m})$} & \multicolumn{3}{|c|}{ Number of SCS's } \\
\hline & & distinct & indistinct & total & distinct & indistinct & total \\
\hline Early & 56 & 139 & - & 139 & 68 & - & 68 \\
\hline diplotene & 65 & 138 & - & 138 & 76 & - & 76 \\
\hline Mid & 51 & 71 & 9 & 80 & 87 & 37 & 124 \\
\hline \multirow[t]{2}{*}{ diplotene } & 54 & 66 & 15 & 81 & 69 & 41 & 110 \\
\hline & 53 & 58 & 2 & 60 & 70 & 9 & 79 \\
\hline Late & 30 & 50 & 15 & 65 & 63 & 20 & 83 \\
\hline diplotene & 40 & 58 & 3 & 61 & 70 & 9 & 79 \\
\hline $\begin{array}{l}\text { Early } \\
\text { diakinesis }\end{array}$ & 66 & 23 & 5 & 28 & 51 & 9 & 60 \\
\hline Mid & 50 & 24 & 10 & 34 & 43 & 30 & 73 \\
\hline diakinesis & 29 & 25 & 5 & 30 & 41 & 14 & 55 \\
\hline $\begin{array}{l}\text { Late } \\
\text { diakinesis }\end{array}$ & 67 & - & - & 29 & - & - & 43 \\
\hline $\begin{array}{l}\text { Late } \\
\text { diakinesis- } \\
\text { prometaphase I }\end{array}$ & 32 & - & - & 29 & - & - & 59 \\
\hline
\end{tabular}


and by the condensed chromatin of the long arm (Figure 4).

A short synaptonemal complex segment is present between the homologous segments of the $\mathrm{X}$ and the $\mathrm{Y}$ chromosomes in most $\mathrm{XY}$ bivalents at diplotene (Table II and Figures 2, 4, 24 and 25). A total of $11 \mathrm{XY}$ bivalents was analyzed of which 7 had a distinct synaptonemal complex segment and only one nucleus lacked a complex segment between the two sex chromosomes (Table II). In two cases, an additional small segment was present in the chromatin of the $\mathrm{X}$ chromosome (Figure 4). Such segments are also occasionally seen in XY bivalents at pachytene in bifurcated regions of lateral components of the $X$ chromsome and do not reflect homologous pairing.

The length of the synaptonemal complex segment between the $\mathrm{X}$ and $\mathrm{Y}$ chromosomes ranges

\section{Table II}

Length of the synaptonemal complex segment (SCS) in $X Y$ bivalents at diplotene and diakinesis.

\begin{tabular}{lcc}
\hline Stage & $\begin{array}{c}\text { Nucleus } \\
\text { number }\end{array}$ & $\begin{array}{c}\text { SCS length } \\
(\mu \mathrm{m})\end{array}$ \\
\hline Early & 65 & 0.2 \\
diplotene & 56 & $-\mathrm{a}$ \\
\hline & 77 & 1.0 \\
Mid & 74 & 0.6 \\
diplotene & 51 & 0.2 \\
& 54 & 0.4 \\
\hline Late & 53 & 0.2 \\
diplotene & 52 & $-\mathrm{b}$ \\
\hline Early & 30 & - \\
diakinesis & 55 & 0.9 \\
\hline Mid & 40 & $-\mathrm{b}$ \\
\hline diakinesis & 66 & 0.7 \\
\hline Late & & - \\
diakinesis & 50 & - \\
\hline Late & 29 & 0.3 \\
diakinesis-prom- & 67 & - \\
etaphase I & & \\
\hline & 32 & - \\
\hline
\end{tabular}

a) Homologous segment of the $X$ and $Y$ chromosomes juxtapositioned.

b) Partially degraded SCS. from 0.2 to $1.0 \mu \mathrm{m}$ with a mean value of $0.5 \mu \mathrm{m}$ (Table II). As shown in Figures 24 and 25, the segment may be present distally or proximally in the short arm of the $\mathrm{Y}$ chromosome.

\subsubsection{Diakinesis - prometaphase I}

The change in the condensation pattern of the $\mathrm{XY}$ bivalent continues during diakinesis, and by late diakinesis the chromatin of the sex bivalent appears morphologically similar to that of the autosomes except for the long arm of the Y chromosome which is distinctly more condensed than the remainder of the XY bivalent and the autosomes (Figures 7, 9,11 and 28). A spherical structure surrounded by an electron transparent shell and associated with remnants of the lateral component of the $\mathrm{X}$ chromosome appears inside the $X Y$ bivalent (Figure 27). The nature and fate of this structure were not investigated further.

At diakinesis, two of the four analyzed nuclei (one early and one mid diakinesis) contained an $\mathrm{XY}$ bivalent with a recognizable synaptonemal complex segment between the sex chromosomes, the mean length amounting to $0.5 \mu \mathrm{m}$ as at diplotene (Table II and Figure 27). In the completely sectioned nucleus at the diakinesis prometaphase I transition, a short synaptonemal complex segment was present inside the chromatin of the $\mathrm{XY}$ bivalent, but due to ambiguities in identifying the centromeric chromatin and the kinetochore of the $\mathrm{Y}$ chromosome it could not be established whether the segment still combined the homologous regions of the $\mathrm{X}$ and $\mathrm{Y}$ chromosomes.

\subsection{The distribution of synaptonemal complex segments among bivalents and bivalent arms at diplotene}

The degradation pattern of synaptonemal complexes described in section 3.3. shows that the resistance of the synaptonemal complex varies along the bivalents. In order to rule out the possibility that the observed pattern of retained synaptonemal complex segments arises as the result of a gradual, progressive degradation which affects all arms simultaneously and at the same rate, the following calculation was carried out: With the mean pachytene complement as a base enough synaptonemal complex was subtracted from all arms to reduce the total complex length 
Table III.

Distribution of synaptonemal complex segments among bivalents at mid-late diplotene and early-mid diakinesis.

\begin{tabular}{lccccccccc}
\hline & \multicolumn{7}{c}{ Percent bivalents with $\mathrm{n}$ synaptonemal complex segments } \\
\cline { 2 - 12 } & 0 & 1 & 2 & 3 & 4 & 5 & 6 & 7 & $>7$ \\
\hline $\begin{array}{l}\text { Mid-late diplotene } \\
\text { (distinct segments) }\end{array}$ & 2 & 8 & 25 & 33 & 13 & 8 & 5 & 5 & 2 \\
\hline $\begin{array}{l}\text { Early-mid diakinesis } \\
\text { (all segments) }\end{array}$ & 5 & 20 & 30 & 24 & 5 & 9 & 5 & 1 & 1 \\
\hline Chiasmata & 0 & 11 & 56 & 26 & 6 & 1 & 0 & 0 & 0 \\
\hline
\end{tabular}

a) $n$ equals the number of chiasmata, data from HULTÉN (11).

Table IV.

Distribution of synaptonemal complex segments among bivalent arms at mid-late diplotene and early-mid diakinesis.

\begin{tabular}{lcccccccc}
\hline & \multicolumn{7}{c}{ Percent bivalent arms with $\mathrm{n}$ synaptonemal complex segments } \\
\cline { 2 - 9 } & $\mathrm{n}=$ & 0 & 1 & 2 & 3 & 4 & 5 \\
\hline $\begin{array}{l}\text { Mid-late diplotene } \\
\text { (distinct segments) }\end{array}$ & 15 & 39 & 28 & 11 & 6 & 2 \\
\hline $\begin{array}{l}\text { Early-mid diakinesis } \\
\text { (all segments) }\end{array}$ & 29 & 29 & 27 & 11 & 5 & 0 \\
\hline Chiasmata & 10 & 64 & 24 & 2 & 0 & 0 \\
\hline
\end{tabular}

a) $n$ equals the number of chiasmata, data from HuLrén (11).

to $63 \mu \mathrm{m}$, which is approximately the same as the mean length at mid-late diplotene. This required the removal of all complex in bivalent arms shorter than $4.5 \mu \mathrm{m}$ and this amount in longer arms. In the determination of the frequency of bivalents without segments, the short arms of acrocentric bivalents were omitted. The calculation revealed that 18 percent of all bivalents and 41 percent of all bivalent arms are in such a case devoid of complex. This result is not consistent with the observation that the $61 \mu \mathrm{m}$ of complex present at mid-late diplotene is distributed so that only two percent of all bivalents and 15 percent of all bivalent arms lack synaptonemal complex segments ( 3 percent if the short arms of the acrocentric bivalents are excluded) (Table III, IV). Degradation of the synaptonemal complex during diplotene is thus regulated at the level of the individual bivalent and bivalent arm, the retention of at least one synaptonemal complex segment in each arm being nearly obligatory. This is also the case for chiasmata at diakinesis as seen in the light microscope, all bivalents and virtually all bivalent arms having at least one chiasma with the exception of the short arms of the acrocentric bivalents (Tables III, IV) and the same pattern has also been inferred for the dis-

Table V.

Distribution of synaptonemal complex segments between the two arms of bivalents with 2 and 3 segments at mid-late diplotene (distinct segments) and earlymid diakinesis (all segments).

\begin{tabular}{lcccc}
\hline & \multicolumn{3}{c}{$\begin{array}{c}\text { Percent segments in arms of } \\
\text { bivalents with } \mathrm{n} \text { segments }\end{array}$} \\
\hline $\mathrm{n}=$ & $(0+2)$ & $(1+1)$ & $(0+3)$ & $(1+2)$ \\
\hline Mid-late diplotene & 39 & 61 & 17 & 83 \\
\hline Early-mid diakinesis & 56 & 44 & 24 & 76 \\
\hline Chiasmataa & 18 & 82 & 2 & 98 \\
\hline
\end{tabular}

a) $n$ equals the number of chiasmata, data from HULTÉN (11) 

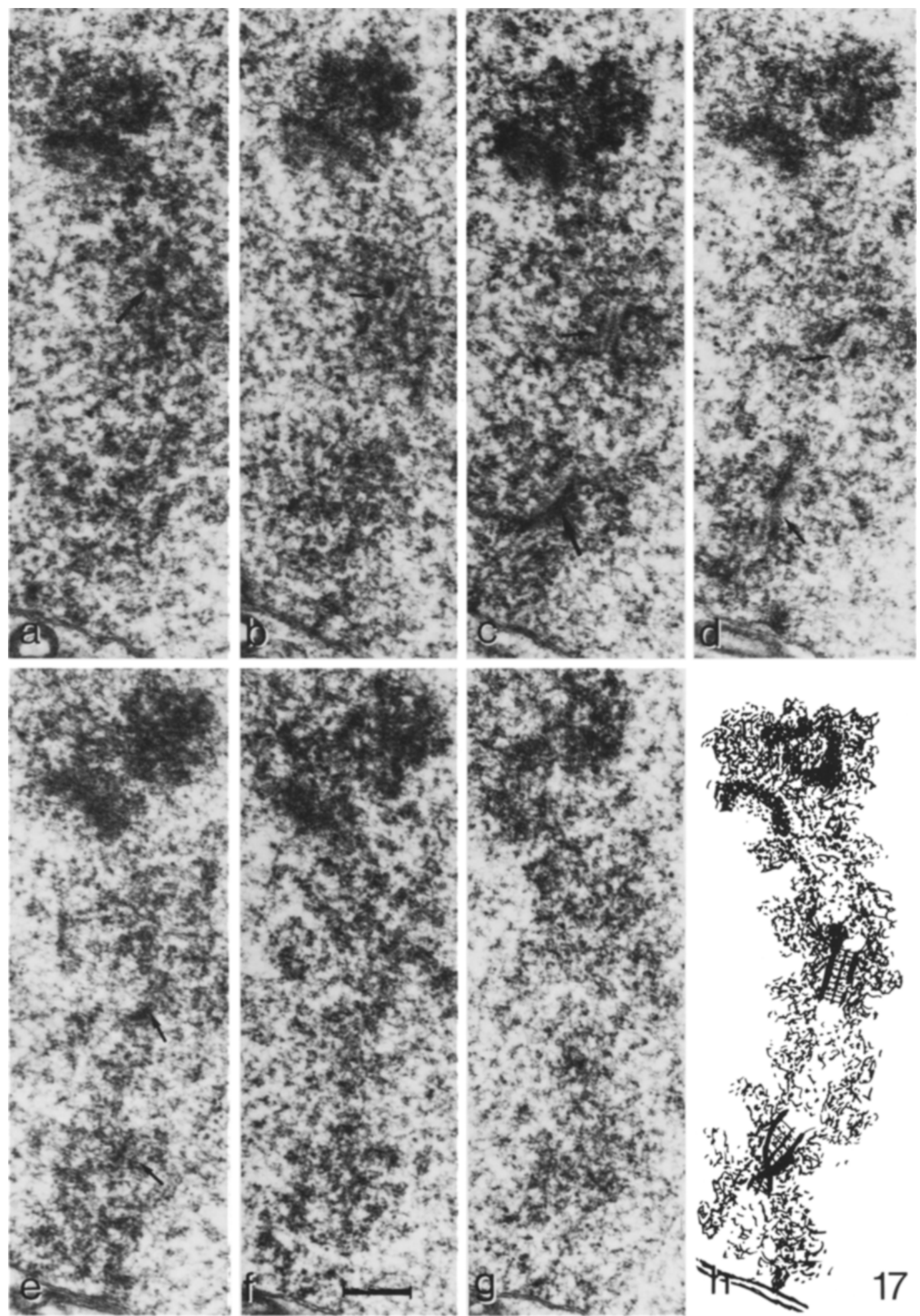
Figure 17. Seven consecutive longitudinal sections through a bivalent arm at late diplotene. Two intact synaptonemal complex segments (denoted by arrows) are present in sections b-e and are shown in the drawing in Figure $h$. The two segments lack nodules and bars and appear ultrastructurally intact with thick dense lateral components and central regions of normal pachytene morphology. A small compact chromatin sphere, confluent with one of the lateral components, is present in both of the segments of the complex as denoted by the large arrows in Figures $a$ and $c$. $(\mathrm{Bar}=0.5 \mu \mathrm{m})$

tribution of recombination nodules and bars at pachytene (10). Furthermore, as shown in Table III, 71 percent of all bivalents at mid-late diplotene contain two, three or four distinct synaptonemal complex segments in agreement with reported numbers of crossovers and chiasmata per bivalent: the D-G group bivalents on the average aquire two, the $C$ group bivalents between two and three and the $A$ and $B$ group bivalents between three and four $(10,11)$. Finally, also the distribution of segments between arms of bivalents with two and three segments resembles that of chiasmata (Table V). This indicates either that the occurrence of a crossover in a bivalent arm simultaneously determines where breakdown of the synaptonemal complex commences after pachytene, or that certain regions of the synaptonemal complex in each bivalent arm both have a high probability for acquiring a nodule/bar and are less susceptible to breakdown at early-mid diplotene.

\subsection{Distribution of synaptonemal complex segments along the bivalents at diplotene}

The aim of this section is to assess whether the position of the retained segments along individual bivalents also resembles the distribution of nodules and bars at pachytene. The diakinesis stage could not be included in the analysis due to ambiguities in determining the absolute length of the bivalents, and one late diplotene nucleus (number 40) was excluded, as the position of several telomeres could not be established with certainty. Hence, the analysis was performed on two early and four mid-late diplotene nuclei. The bivalents within some of the Denver groups could not be distinguished unambiguously, and the bivalents were therefore grouped as follows: 1, 2, 3, 4-5, 6-12 (minus 9), 9, 13-15, 16-18, 19-20 and $21-22$. The lengths of the bivalent arms were then normalized to the mean length of the group, divided into $0.67 \mu \mathrm{m}$ intervals and the frequency of distinct synaptonemal complex determined for each interval. In order to facilitate the interpretation, the histograms were transformed into curves by plotting the mean frequencies of two neighbour intervals, the only interval represented by its actual frequency being the telomeric one (Figure 28).

Considering the small number of nuclei upon which the diagrams in Figure 28 are based, there is a remarkable resemblance between the distribution of paired regions along the bivalent arms at early and mid-late diplotene. The distribution of peaks and troughs seems to follow similar patterns in all bivalent arms at both periods: (1) There is always a trough in the centromere region and a peak near the telomere. (2) If the length exceeds the mean length of bivalents 16-18 (i.e., about $4 \mu \mathrm{m}$ ), the arm contains one or more additional peaks, the distance between the peaks ranging from $2-4 \mu \mathrm{m}$.

The observations show that the susceptibility of the synaptonemal complex to degradation varies in a characteristic way along the bivalent arms, regions of high susceptibility alternating with more resistant regions. Furthermore, the patterns are very similar at early and mid-late diplotene.

As discussed in the previous paper (10), the combined frequency per interval of bars at pachytene stage 2 and of nodules/bars at stages 3-7 constitute the best approximation to the distribution of total crossover events. The pachytene bivalent arms were grouped and normalized to the respective mean diplotene length. As for the diplotene bivalents, the frequencies plotted in the diagram are the mean of a given interval and one neighbour interval. The frequencies were calculated as the number of nodules/bars per interval assuming a mean number of 50 nodules/bars per nucleus. A comparison between the distributions of nodules/bars at pachytene and distinct synaptonemal complex segments at mid-late diplotene shows that the two distributions share general features (Figure 


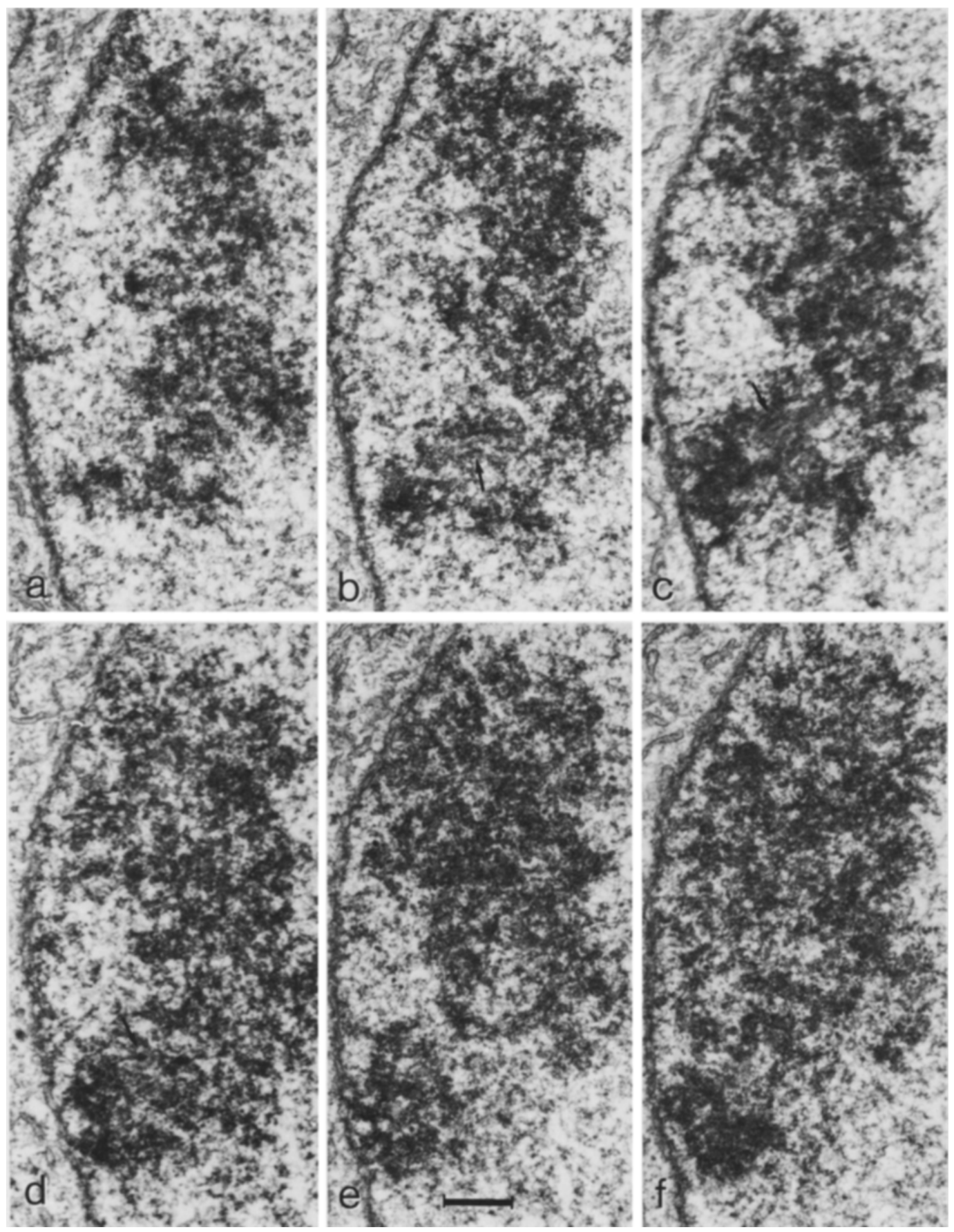



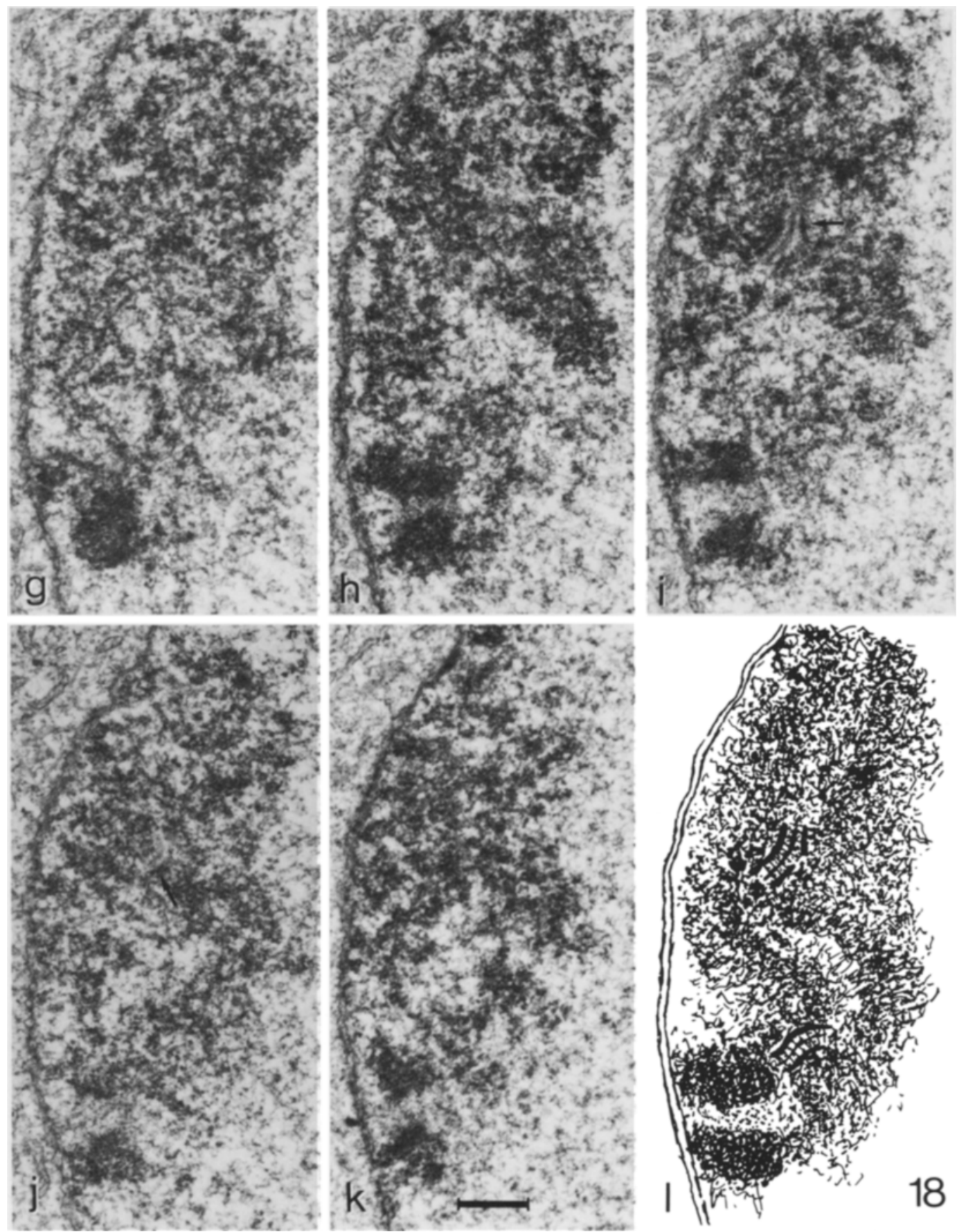

Figure 18. Eleven consecutive longitudinal sections through an acrocentric bivalent at early diakinesis. The series encompasses the entire central part of the bivalent including the confluent centromere regions and the prokinetochores. Two distinct synaptonemal complex segments (denoted by arrows) are present in the central region of the bivalent, one in the vicinity of the centromere (sections b-d), the other in the middle of the long arm (sections $\mathrm{i}-\mathrm{j}$ ). Ultrastructurally, the complex segments appear normal as do their association with the homologous chromosomes. $($ Bar $=0.5 \mu \mathrm{m})$ 

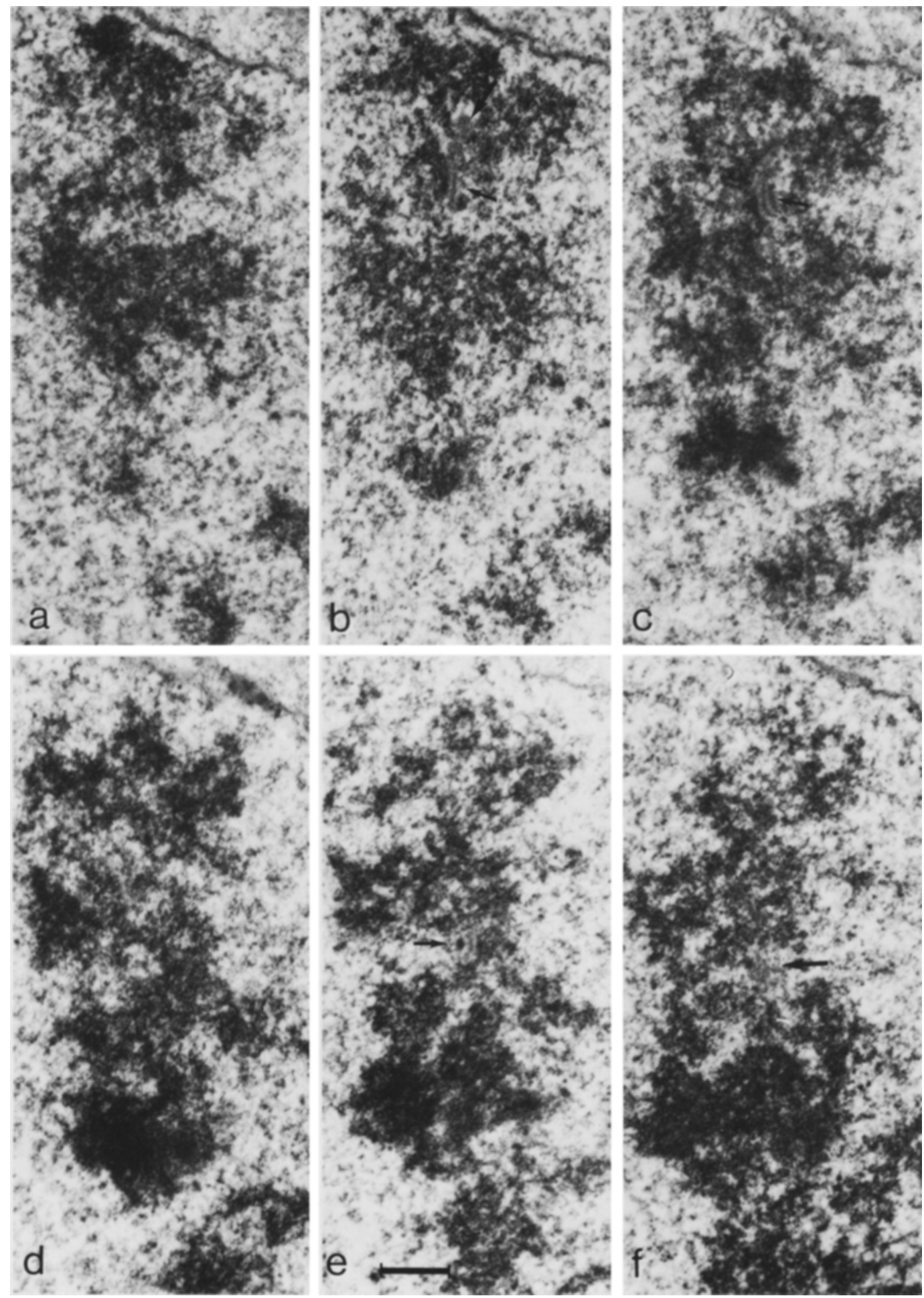

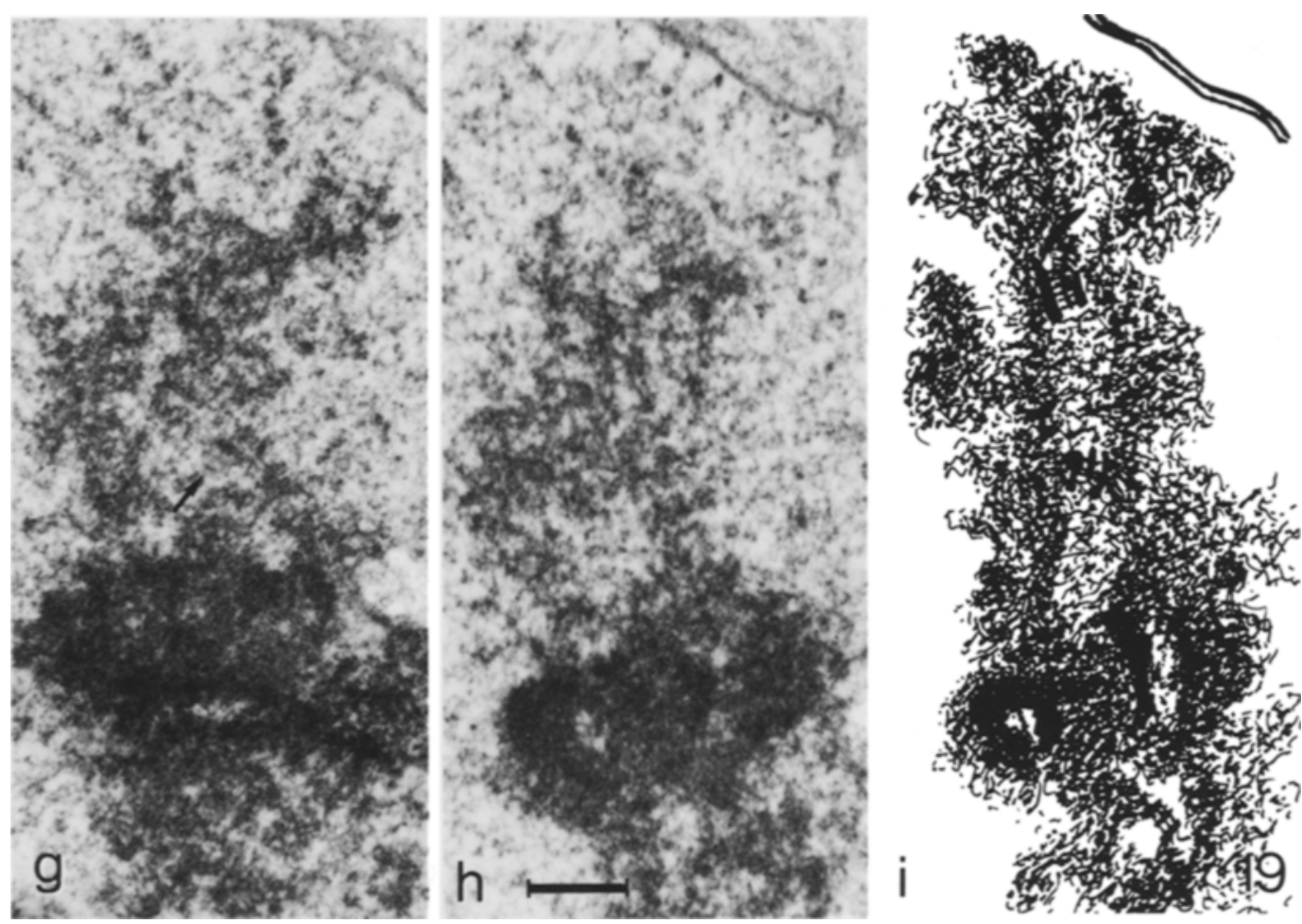

Figure 19. Eight consecutive longitudinal sections through the long arm of bivalent 1 at mid diakinesis. Two synaptonemal complex segments (denoted by small arrows) are present in this series, both located in the central axis of the bivalent. In contrast to the situation at earlier stages, the integrity of the complex segments appears affected, disorganized central region constituents having accumulated at one end of each of the segments as denoted by the large arrows in Figures $b$ and $\mathrm{f} .(\mathrm{Bar}=0.5 \mu \mathrm{m})$

28): (1) There is, in both distributions, a distinct trough in the centromere region and a peak near the telomere region, (2) the position and number of peaks and troughs in the bivalent arms are approximately the same, the number of peaks being roughly proportional to the length of the arm. Minor differences are, however, also evident: (1) The distal peaks of the nodule/bar distribution are often closer to the telomere than those of the distinct segments at mid-late diplotene, (2) the telomeric peaks of the nodule/ bar distribution are in all bivalents somewhat larger than the interstitial peaks, whereas this is not to the same extent the case for the distribution of distinct segments at mid-late diplotene.

These observations indicate that the early synaptonemal complex degradation is initiated in regions which at pachytene have the lowest prob- ability of crossing over and is retarded in regions with a high probability of crossing over.

\subsection{Distribution of synaptonemal complex segments at diakinesis}

As most of the synaptonemal complex segments at diplotene probably include regions where crossing over has occurred, the same is expected for the segments at early to mid diakinesis. This argument is also supported by the number of segments and by the presence of chromatin bridges in the vicinity of some of the segments at late diakinesis. Crossing over at pachytene may thus result in a second modification of the synaptonemal complex in the immediate vicinity of the site of crossing over which makes this region more resistant to degradation so that these synaptonemal complex segments 

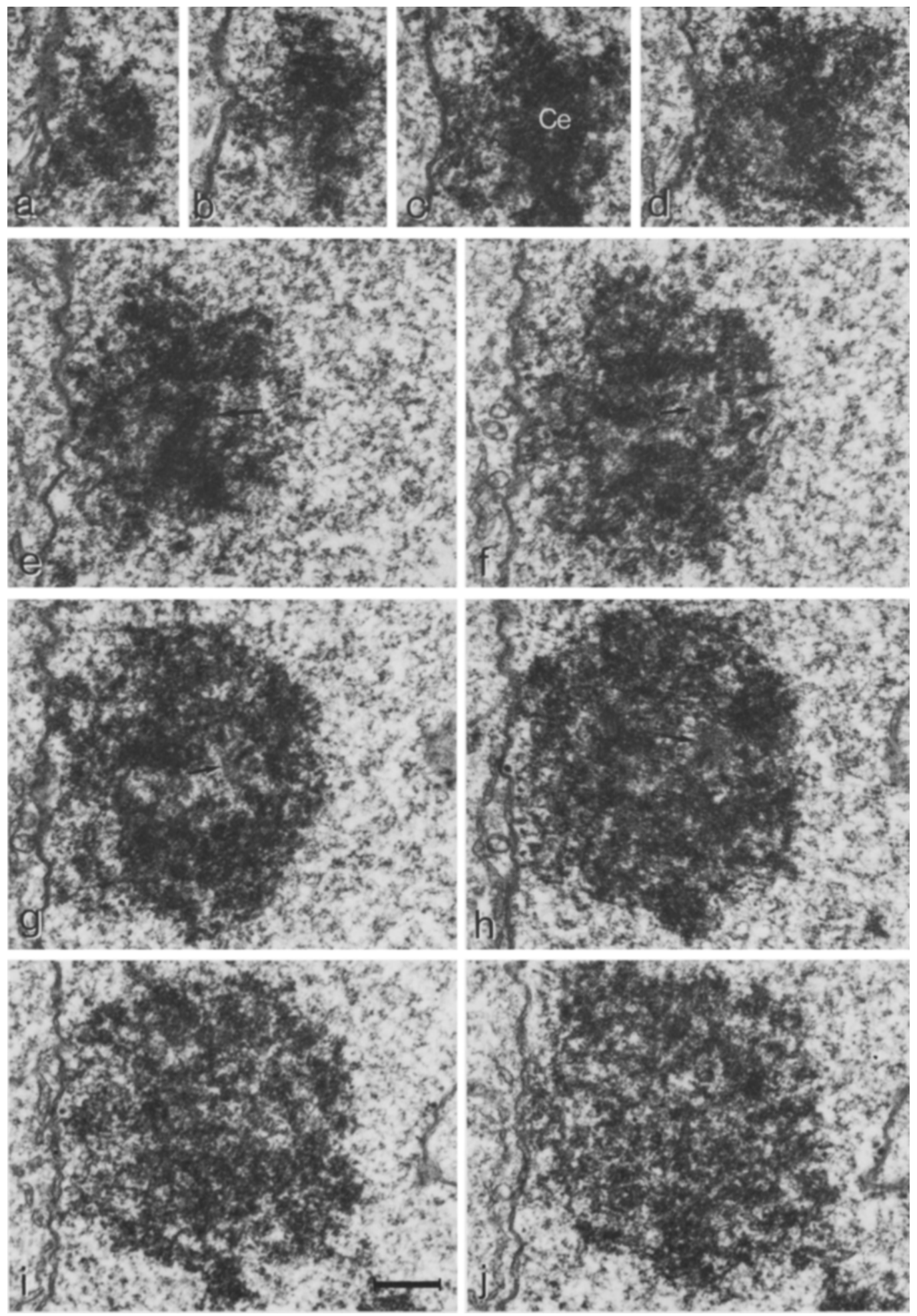

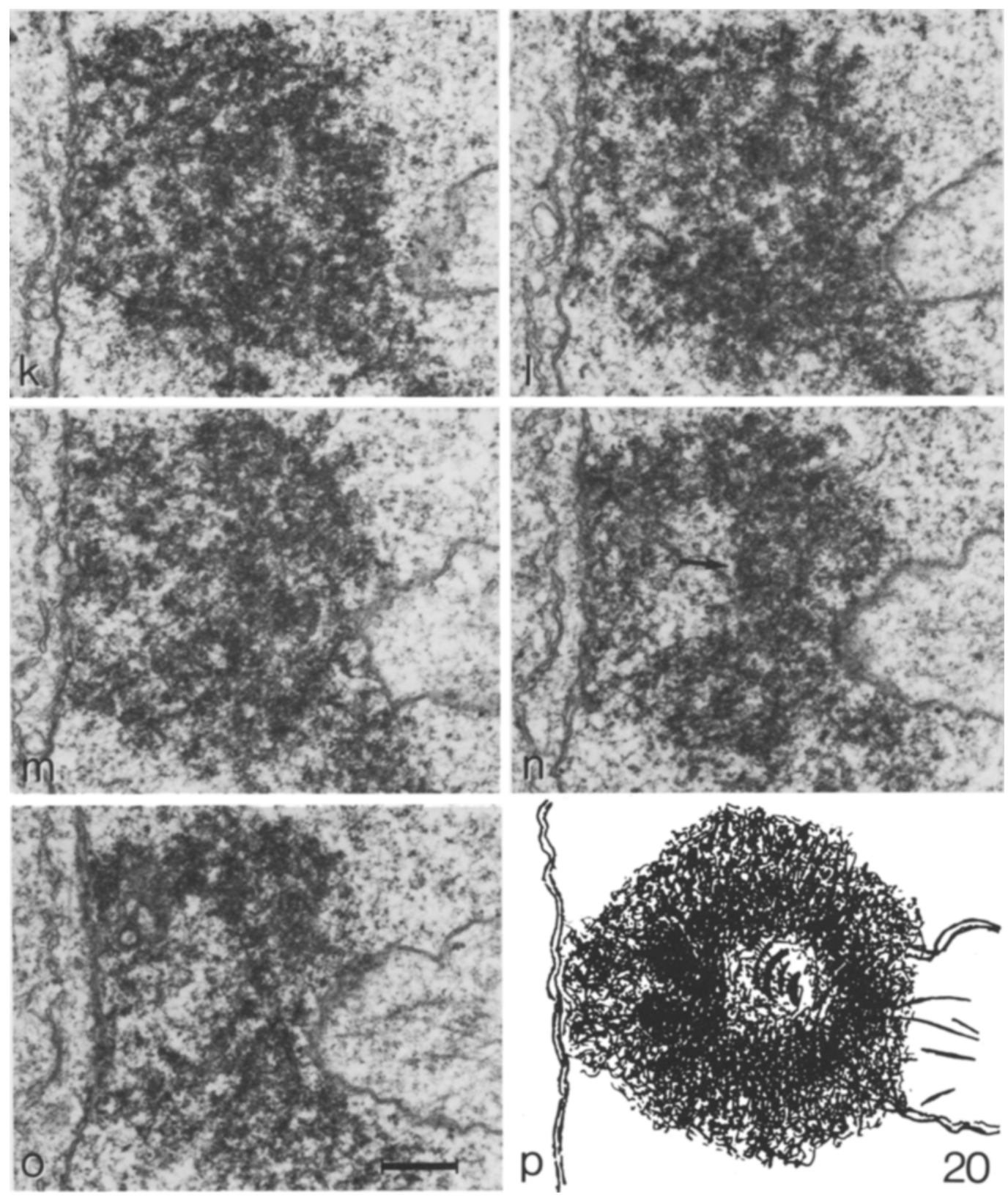

Figure 20. Fifteen consecutive sections through an acrocentric bivalent at late diakinesis. The bivalent is cut in almost exact cross section, the confluent regions of centromeric chromatin of the two homologues being included in sections a- $d$ and the two prokinetochores in section $d$. An interpretative drawing of the bivalent is shown in Figure $\mathrm{p}$. Remnants of the synaptonemal complex, denoted by arrows in sections $\mathrm{f}-\mathrm{h}$, have fused into a small aggregate of medium dense central region material and dense fragments of lateral components. The remnants appear disorganized without well-defined associations to the chromatin. The two homologues are in all sections entirely or partially confluent and it is at most levels not possible to delimit the individual chromosomes. Structurally well-defined chiasmata are not apparent, the most presumptive regions of exchange being those indicated by the large arrows in Figures $\mathrm{e}$ and $\mathbf{n}$. Throughout its length, the bivalent is in contact with the nuclear envelope. (The membrane bound region in Figures $\mathrm{g}-\mathrm{o}$ is one of the two indentations of the nuclear envelope in which the centriole pairs are located, cf. Figure 11). $($ Bar $=0.5 \mu \mathrm{m})$ 


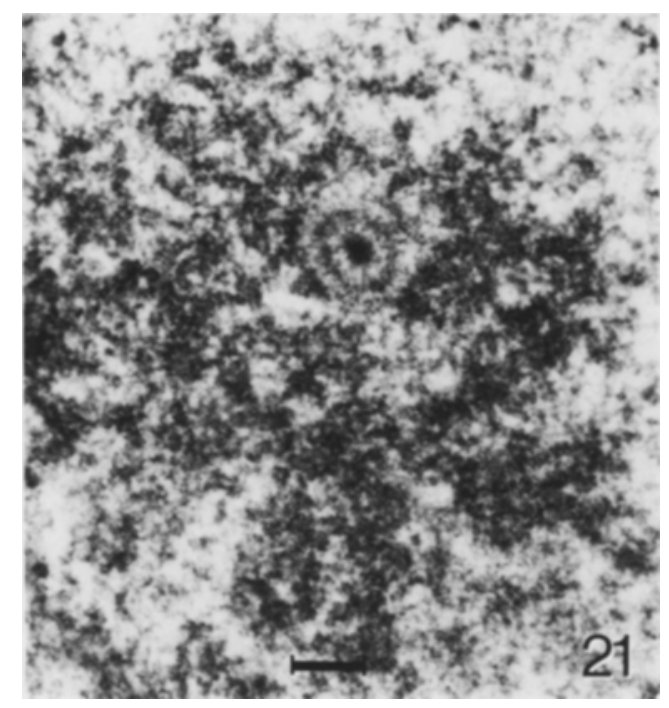

Figure 21. Circular synaptonemal complex segment inside the chromatin of a bivalent at late diakinesis. $(\mathrm{Bar}=0.2 \mu \mathrm{m})$

Figure 22. Three consecutive sections through a late diakinesis bivalent showing a presumptive chiasma (C) next to a partially degraded synaptonemal complex segment (denoted by arrows). $(\operatorname{Bar}=0.5 \mu \mathrm{m})$

Figure 23. Histograms showing the distribution of synaptonemal complex segment sizes at mid-late diplotene (hatched area) and early-mid diplotene. Each size class of $0.1 \mu \mathrm{m}$ represents the mean value of that particular class, and the two adjacent ones on either side.
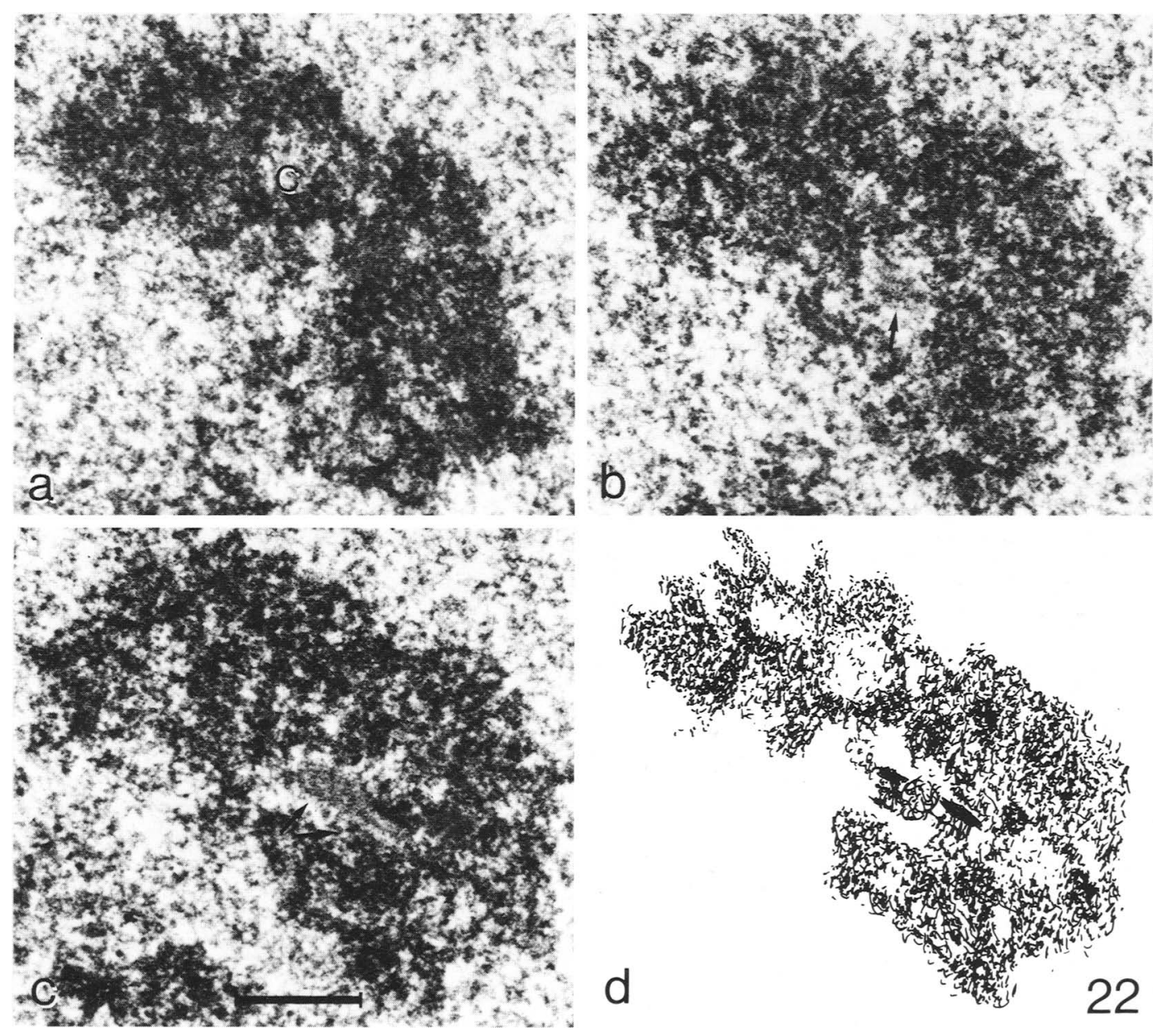
P.B. Holm \& S.W. RaSmuSSEN: Human meiosis VII

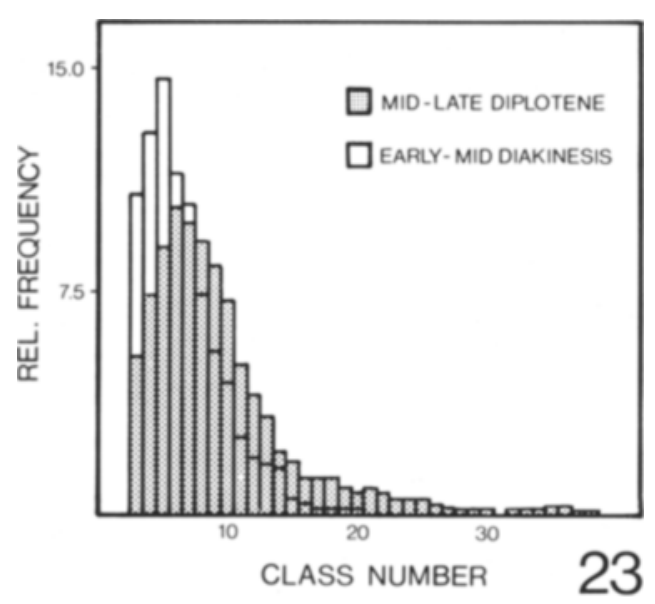

Figure 24. Five consecutive sections through an $X Y$ bivalent at early diplotene. The centromeric chromatin of two autosomal bivalents (A) are associated with the $X Y$ bivalent as shown in the drawing, Figure f. A short segment of intact synaptonemal complex (denoted by an arrow in Figures c-e) is present in the confluent chromatin of the $\mathrm{X}$ and $\mathrm{Y}$ chromosomes attached to the nuclear envelope. The thick lateral component of the $Y$ chromosome reaches the centromere region. The unpaired parts of both lateral components are abruptly bent and are interconnected in the region near the centromere of the $\mathrm{Y}$ chromosome. $\mathrm{Ce}, \mathrm{Y}$ centromere region. $(\mathrm{Bar}=0.5 \mu \mathrm{m})$
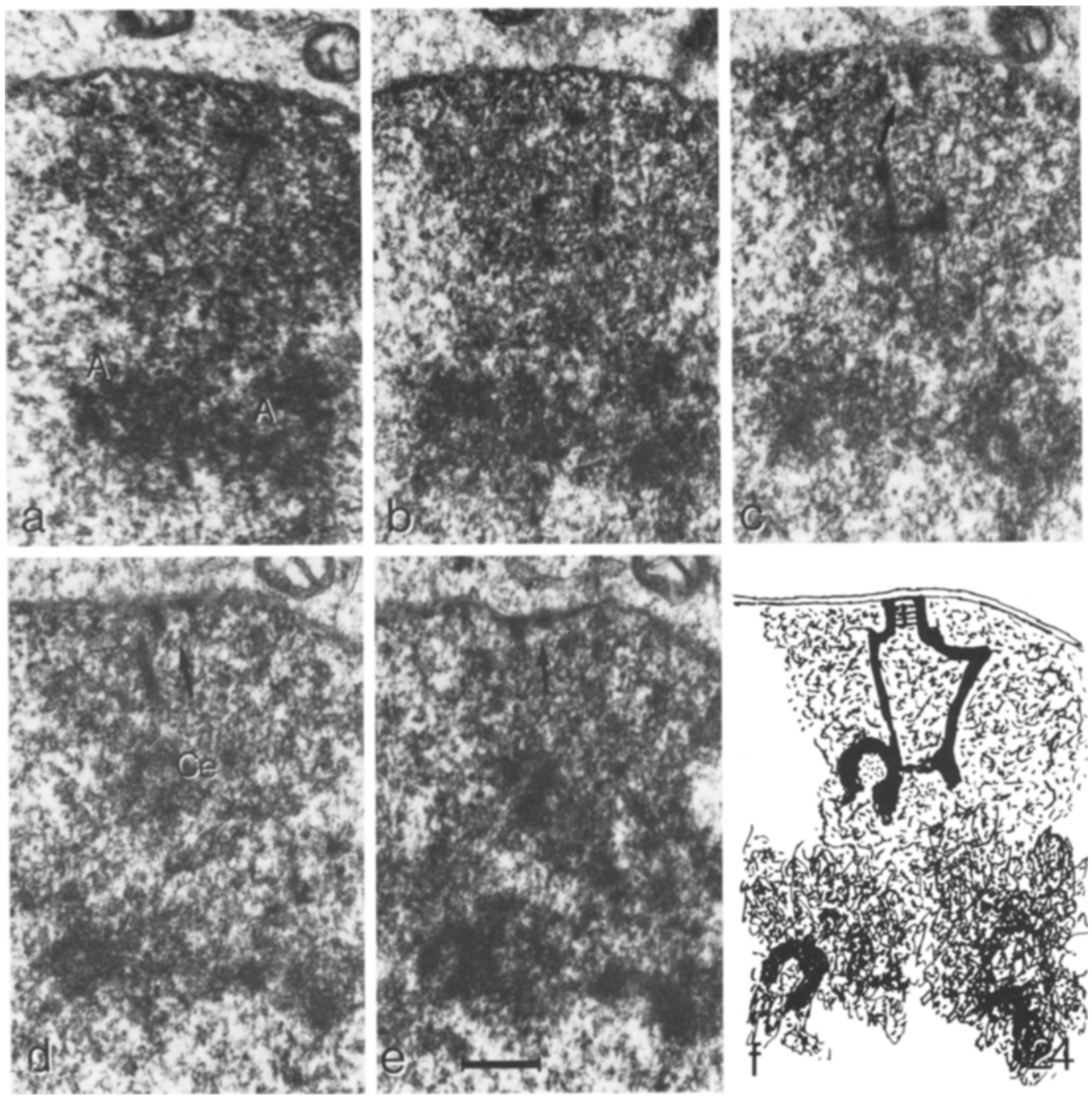

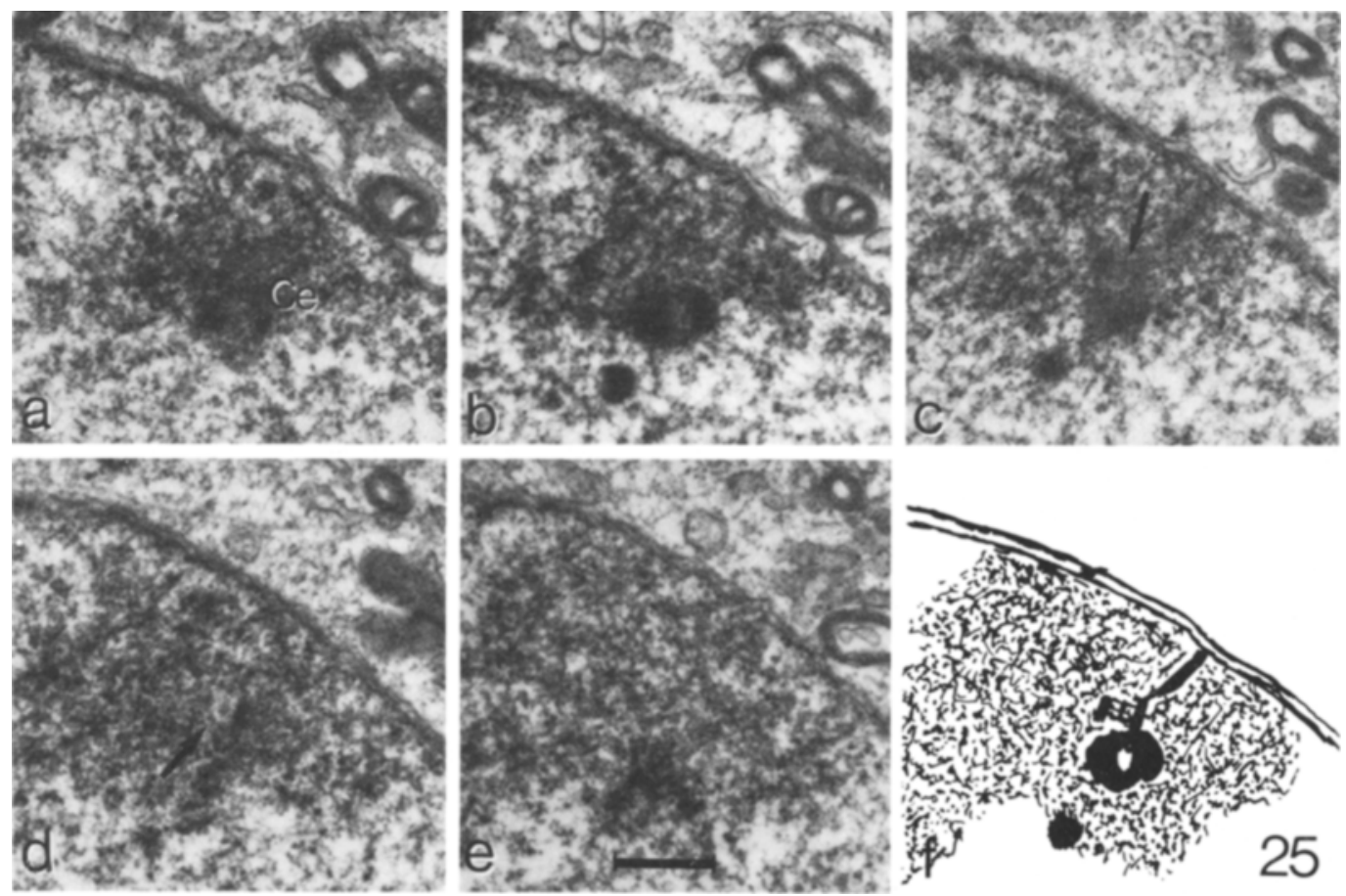

Figure 25. Five consecutive sections through an XY bivalent at mid diplotene. As shown in the drawing, Figure f, the lateral component of the $\mathrm{Y}$ chromosome is intact from its attachment site on the nuclear envelope to the centromere region. A short segment of intact synaptonemal complex is denoted by arrows in Figures $\mathrm{c}$ and $\mathrm{d}$, the segment in the former figure being filled with dense material. $\mathrm{Ce}, \mathrm{Y}$ centromere region. (Bar $=0.5 \mu \mathrm{m}$ )

are retained up to late diakinesis and first completely eliminated at metaphase I. However, as shown in Table III, five percent of the bivalents at early-mid diakinesis lack a synaptonemal complex segment and 20 percent have only one, the corresponding frequencies for mid-late diplotene being two and eight percent. Elimination of some synaptonemal complex segments associated with crossovers may thus occur already at or even before early diakinesis. The data in Table IV suggest that the segments primarily are eliminated from arms which only contain a single segment as the frequency of bivalent arms with one segment decreases from 39 percent at mid-late diplotene to 29 percent at early-mid diakinesis, while bivalent arms with two or more segments are present with the same frequency. In agreement with this, the frequency of bivalents where all segments are contained in the same arm has increased at early-mid diakinesis relative to the frequency of bivalents in which both arms contain synaptonemal complex segments. Hence, an early elimination of a synaptonemal complex segment may primarily occur in bivalent arms with only one segment at mid-late diplotene.

\section{DISCUSSION}

\subsection{Diplotene and diakinesis in human spermatocytes}

The present investigation has shown that the diplotene and diakinesis stages of human spermatogenesis are of short duration compared to other prophase stages as judged by the rare occurrence of nuclei at these stages in the seminiferous tubules and by the presence of cells ranging from diplotene to prometaphase I within the same cluster. By these criteria, the diplotenediakinesis period is even shorter than the prometaphase-metaphase interval.

Apart from light microscopical analyses of 

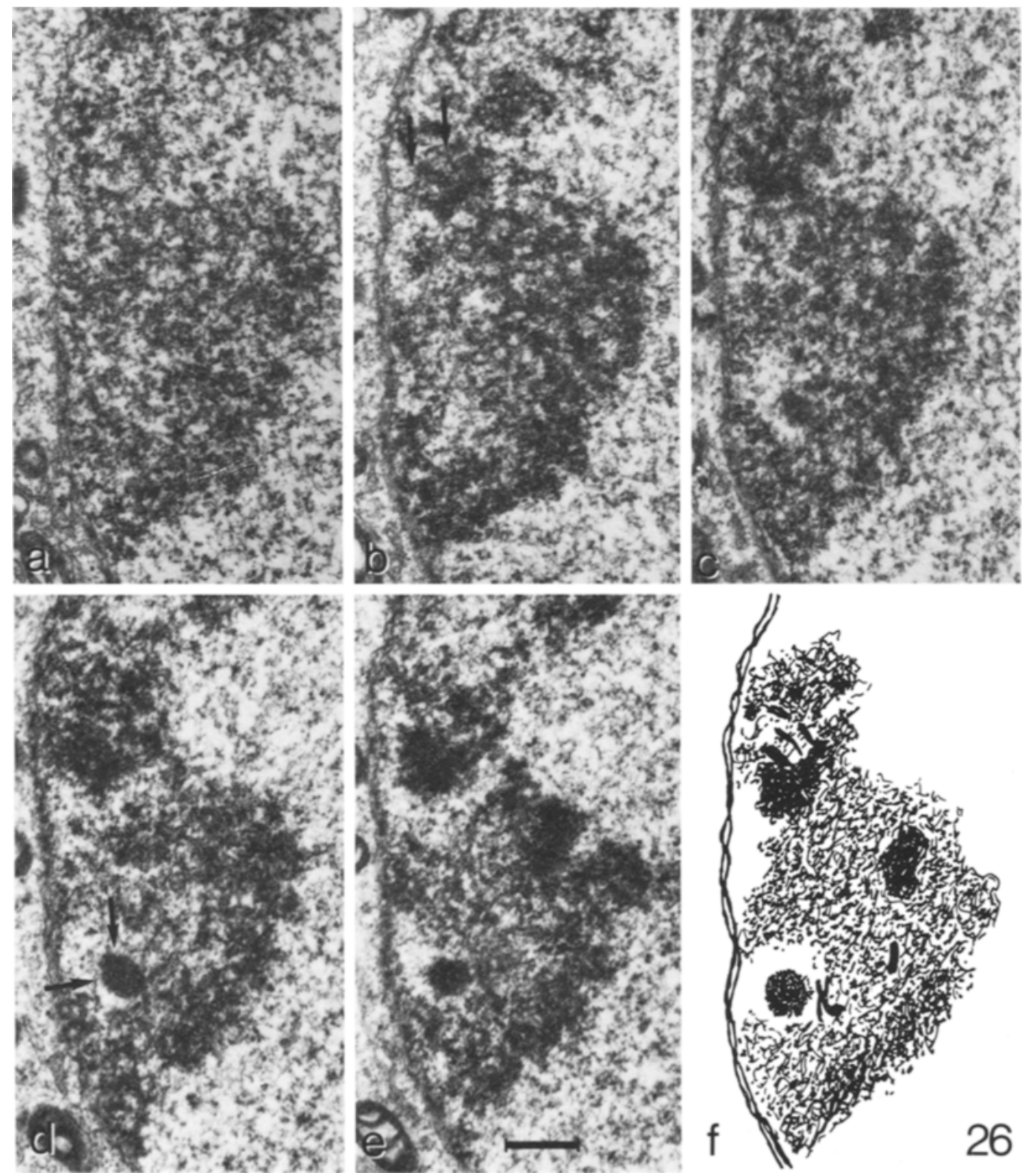

Figure 26. Five consecutive sections through an XY bivalent at early diakinesis. Remnants of the synaptonemal complex are present in section $b$ (arrows) close to the centromere region of the $Y$ chromosome. A dense spherical structure is associated with remnants of the lateral component of the $\mathrm{X}$ chromosome (arrows in Figure $\mathrm{d}$ ). (Bar = $0.5 \mu \mathrm{m})$

chiasma distributions at diakinesis to be discussed later, the available information on the diplotene-diakinesis period is scanty. Recently, SoLARI (22) reported that diplotene nuclei microspread for electron microscopy are large, have diffuse chromatin and contain only few fragments of lateral components of the synaptonemal complex. This description is not consistent with the present observations and the nuclei described by SOLARI must either have been severely 

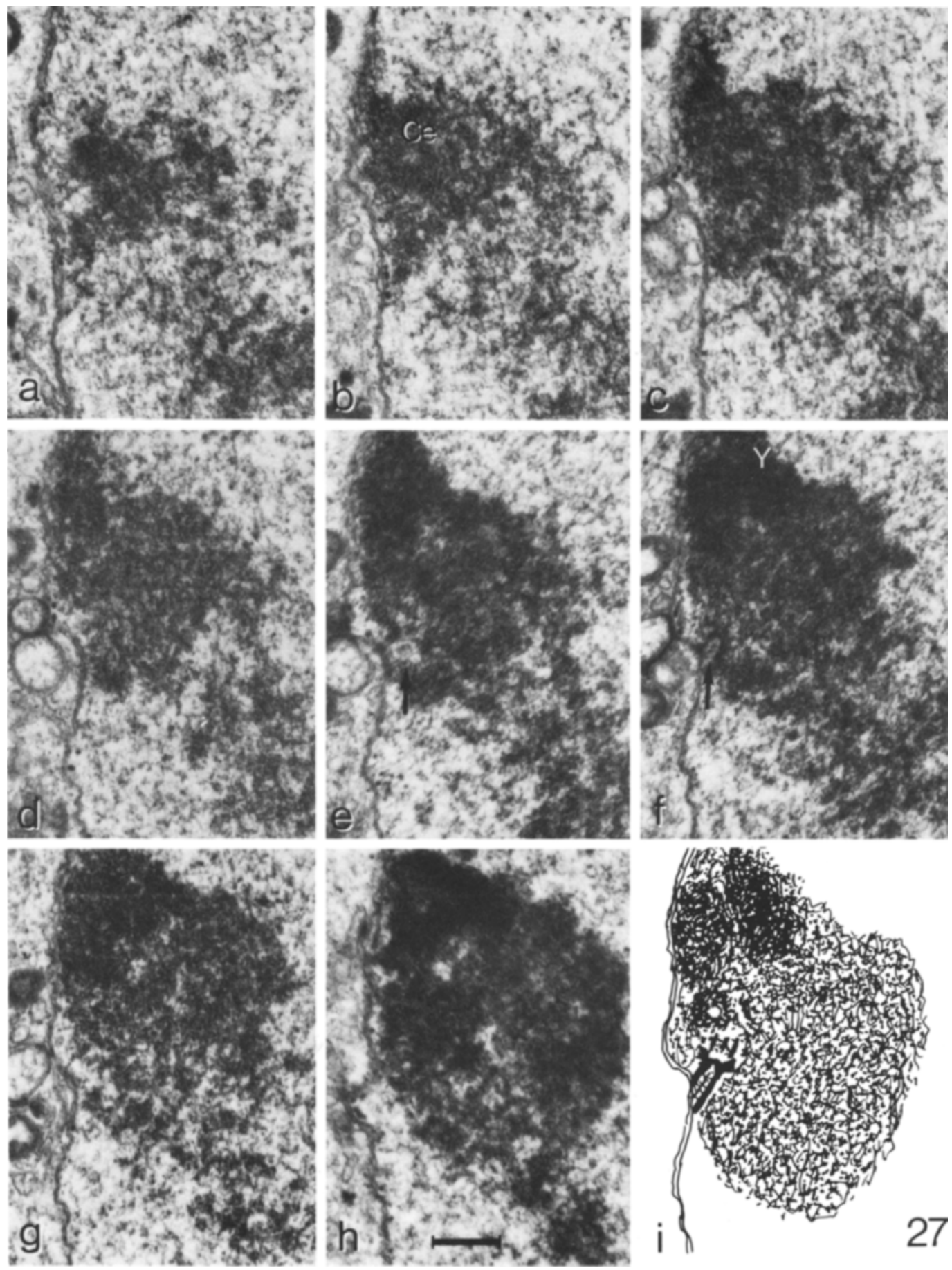

Figure 27. Eight consecutive sections through an $\mathrm{XY}$ bivalent at early-mid diakinesis. A reconstruction is shown in Figure 27i. An intact synaptonemal complex (denoted by arrows) is attached to the nuclear envelope in sections e-f and is at the other end associated with dense material bridging the central region of the complex. The synaptonemal complex segment appears to continue through sections $\mathbf{c}$ and $\mathbf{d}$ terminating close to the centromere region of the $\mathrm{Y}$ chromosome. The long arm of the $\mathrm{Y}$ chromosome $(\mathrm{Y})$ has condensed into a compact body. $(\mathrm{Bar}=0.5 \mu \mathrm{m})$ 

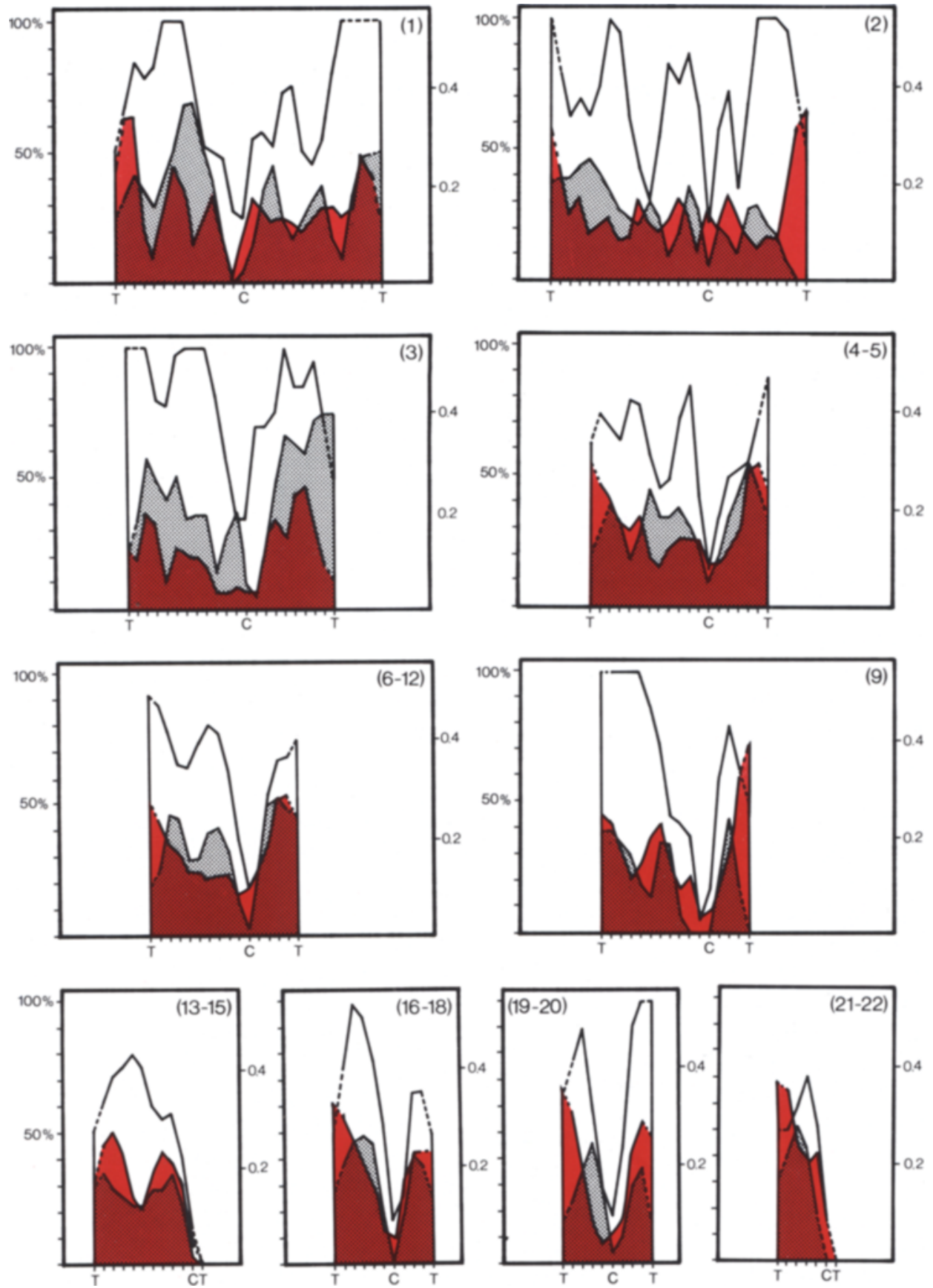

Figure 28. Frequency distribution of synaptonemal complex fragments along bivalents $1,2,3,4-5,9,6-12$ (minus 9), 13-15, 16-18, 19-20 and 21-22 at early (no hatching) and mid-late diplotene (light hatching). The crossover distribution obtained through the analysis of recombination nodules at pachytene (10) is shown in red, the total frequency of crossovers being corrected to a mean of 50 per nucleus. On the $\mathrm{X}$-axis is shown the mean length of the bivalents with $\mathrm{T}$ and $\mathrm{C}$ denoting the position of the centromere and the telomere, the short arm lying to the right. The left $Y$-axis refers to the mean frequency of synaptonemal complexes present and the right $Y$-axis the number of crossovers per nucleus and segment. The frequencies plotted in the diagram are the mean of the frequency in each interval (length $0.67 \mu \mathrm{m}$ ) and one neighbour interval, the only intervals represented by their actual frequencies being the telomeric ones (shown with a broken line). 
damaged by the microspreading procedure, have been degrading or have been at early leptotene.

In spite of the short duration of the period, the analysis of 12 completely and 5 partially reconstructed nuclei revealed a continuous series of morphological changes which warranted a subdivision of the period into well defined substages. In general, diplotene may be defined as the period where the nuclei are spherical, the centrioles are in the vicinity of each other and the length of the complement approximately the same as in pachytene, while a subdivision of this stage is primarily based on the degree of synaptonemal complex elimination. The beginning of diakinesis is marked by a pronounced bivalent shortening and chromatin condensation and the bivalents can be traced by their chromatin contour. The nuclei become irregular and the two pairs of centrioles are widely separated. During the last part of diakinesis, the remaining synaptonemal complex segments are shed from the bivalents but are not degraded. The bivalents are compact but still separate and the nuclear envelope is continuous but of irregular shape with the two centriole pairs located within indentations of the nuclear envelope. Thereafter, the breakdown of the nuclear envelope commences and the bivalents fuse into confluent chromatin masses and all synaptonemal complex segments are present as polycomplexes of varying shape and size as described previously (19). These correlated changes in chromatin contraction, nuclear shape and centriolar behaviour agree well with that observed during diakinesis in Bombyx spermatocytes (7). Furthermore, the late diakinesis stage is in both organisms characterized by a change in chiasma structure, in Bombyx by the elimination of the circular component from the chromatin chiasma and in human spermatocytes by the elimination of the synaptonemal complex segment.

\subsection{The elimination of the synaptonemal complex}

The elimination of the synaptonemal complex from the bivalents and its subsequent degradation is in the human male a regulated process with the following characteristics: 1) Synaptonemal complex degradation is initiated at a low number of sites, the number of remaining synap- tonemal complex segments being the same as that of crossovers but exceeding the number of chiasmata ( 72 segments versus 69 crossovers (10) and 50 chiasmata (11)). The distribution of the resultant stretches among and along the bivalent complement is very similar to the frequency and distribution of crossing over among and along the complement (10). 2) The distribution of regions resistant and susceptible towards degradation along the bivalents appears to be basically similar in all the analysed diplotene nuclei. 3) An apparently constant fraction of the synaptonemal complex is retained during diakinesis. The number of these segments (mean of 62) is slightly less than the number of crossovers but is in excess of the number of chiasmata counted in the light microscope (11).

Together, these observations suggest that the pattern emerging after the initial breakdown of the complex reflects an inherent property of the chromosome/bivalent arm which somehow affects the structure/composition of the synaptonemal complex but also, during mid-late pachytene, results in a nonuniform probability of crossing over along the bivalent arm: Regions of the complex which have the lowest probability of crossing over are first degraded whereas the stretches with the highest probability are those remaining at mid-late diplotene. The degradation pattern furthermore indicates that the crossing over event itself introduces a more local structural modification of the complex which causes a further delay of its shedding from the bivalents and postpones the complete degradation of the constituents of the complex until metaphase-anaphase I (see Figure 29 for a diagrammatic presentation).

This conclusion is in agreement with the hypothesis emerging from the study of recombination nodules and bars at pachytene (10) that the number and distribution of crossovers primarily is a function of inherent domains along the synaptonemal complex of the bivalent arms within which the binding affinity for further nodules is reduced following the first crossover.

Temporary retention of synaptonemal complex segments after pachytene was first observed in the ascomycete Neottiella by WESTERGAARD and VON WETTSTEIN $(25,26)$ who suggested that segments of the complex were retained at sites 
where crossing over had occurred and were holding the repulsing homologues together. Later, at diakinesis, the synaptonemal complex segments disappeared and more stable chromatin connections were assumed to maintain the association between the homologous chromosomes. The same conclusion was reached by SOLARI (21) in a study of mouse spermatocytes. At diplotene, converging segments of lateral components were held together at some sites by a short piece of central region. Subsequently, the retained segments were replaced by chromatin connections.

Retained synaptonemal complex segments have furthermore been observed at diplotene in Zea mays (4), Sordaria macrospora (27), Neurospora crassa (5) and in Ascaris suum (14). In the latter three species, recombination nodules were found in most of the retained pieces. Three dimensional reconstructions revealed in Sordaria and Neurospora a number of segments about equal to the number of chiasmata/ crossovers. In all three species, most or all segments disappeared before diakinesis.

Hence, in these organisms synaptonemal complex segments appear to be retained at sites where crossing over has occurred. Furthermore, in Sordaria (27) and Neurospora (5) approximately 20 percent of all segments at diplotene were devoid of recombination nodules and in Zea (4) the number of retained segments at diplotene exceeded that of chiasmata by a factor of two. Also in Bombyx spermatocytes (7) and in Coprinus meiocytes, segments of synaptonemal complex occasionally appeared to be devoid of recombination nodules or their derivatives.

These data are consistent with the observations in human spermatocytes and suggest that the initial degradation pattern of the synaptonemal complex also in other organisms reflects inherent properties of the bivalents rather than the actual distribution of crossovers.

\subsection{Ultrastructure of chiasmata}

In human spermatocytes, it has not been possible to demonstrate a continuous development of ultrastructurally distinguishable chiasmata from bivalent regions which at pachytene contain recombination nodules or bars. However, as outlined in the preceeding section a number of observations strongly indicate that the crossovers are housed in the distinct synaptonemal complex segments remaining during diplotene and diakinesis. These segments appear to be intimately associated with the chromatin of both homologous chromosomes up to early diakinesis. Thereafter, the segments are gradually shed from the bivalents and replaced by chromatin bridges and by late diakinesis only few are still associated with the homologues.

Analysis by serial sectioning and three dimensional reconstruction of the entire diplotenemetaphase I period in the male silkworm, Bombyx mori (7) and the diplotene-diakinesis period in the basidiomycete, Coprinus cinereus (8) have shown that chromatin-like derivatives of recombination nodules (in Bombyx termed chromatin nodules) are associated with synaptonemal complex fragments. In Coprinus, the retained segments often extend over the whole arm, while shorter fragments or lateral components alone are present in Bombyx. By late diplotene, these remnants are eliminated and the homologues held together by chromatin bridges - chiasmata which in Bombyx consist of two compact chromatin regions, one on each homologue, combined by a chromatin bridge containing a circular structure. The chromatin chiasma in Coprinus also contains a circular structure but of smaller diameter than in Bombyx. The circular structures disappear by late diakinesis in both organisms and by metaphase I only few remain.

The reported differences in the role of the synaptonemal complex in chiasma formation show that interspecific modifications/variations exist and that factors like the relative duration of the prophase stages (the pachytene stage is long and the diplotene-diakinesis period is extremely short in the human male) may lead to apparent differences in the behaviour of the synaptonemal complex after pachytene. The general rule in the analyzed organisms seems, however, to be that segments of the synaptonemal complex are required transitorily in chiasma formation.

\subsection{The $X Y$ bivalent}

A short piece of distinct or partially degraded synaptonemal complex was present between the $\mathrm{X}$ and $\mathrm{Y}$ chromosomes in six out of the seven early and mid diplotene nuclei, while the homol- 

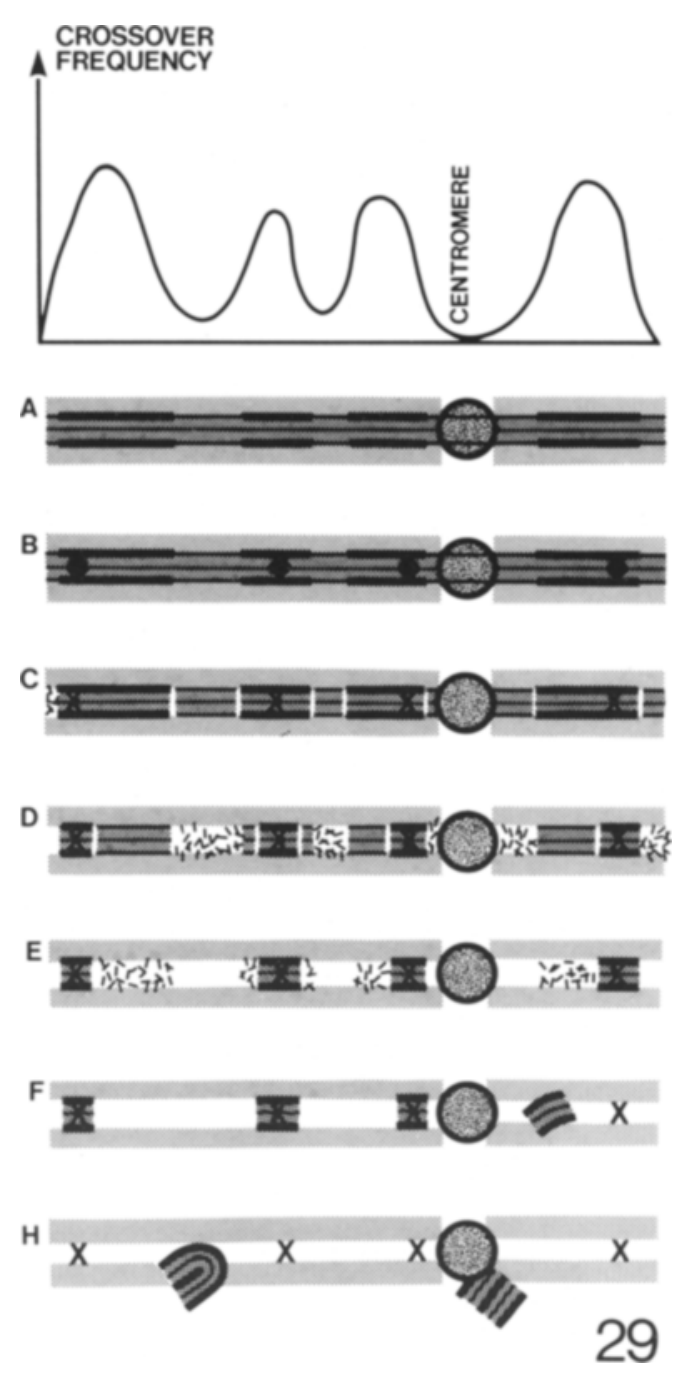

Figure 29. Schematic illustration of synaptonemal complex elimination in a bivalent during diplotenemetaphase I. A-B, pachytene; C-D, diplotene; E-F, diakinesis; $\mathrm{H}$, diakinesis-promataphase $\mathrm{I}$.

ogous segments were juxtapositioned in the last nucleus. As 73 and 75 percent of the XY bivalents at pachytene stages $I$ and 2 contain one or two recombination nodules, the observed frequency of synaptonemal complex segments at early and mid diplotene is consistent with the proposal that the bivalent arms contain a segment of complex with a high affinity for recombination nodules and a higher resistance towards degradation. Furthermore, synaptonemal complex segments are retained in 75 percent of the late diplotene nuclei and 50 percent of the diakinesis nuclei which shows that crossing over has actually occurred in these XY bivalents. At both stages, degradation is in progress suggesting that elimination of the synaptonemal complex segment is initiated fairly early in the $\mathrm{XY}$ bivalent.

\subsection{Conformation of bivalents during diplotene and diakinesis}

As described in section 3.3, the two homologues of all autosomal bivalents remain aligned throughout their length during diplotene and early-mid diakinesis. Even at late diakinesis the two centromere regions of only three bivalents had separated in the completely reconstructed nucleus, the remainder of the homologues still being aligned. The intimate association of the homologous chromosomes, in particular the regions of centromeric chromatin, is even more pronounced after the reduction in nuclear volume and condensation of the bivalents at late diakinesis-prometaphase. Even at prometaphase, the homologous centromeres were confluent in all but one bivalent of the earliest of the three previously reconstructed nuclei (19).

Reconstructions of diplotene bivalents of maize (4) did not reveal separation of homologous regions after shedding of the synaptonemal complex and also in Bombyx, the homologues remain aligned throughout their length during diakinesis (7). Although complete reconstructions of diplotene and diakinesis bivalents could not be performed in diploid Coprinus meiocytes (8), the analysis showed that homologous centromere regions remain closely associated from the end of zygotene at least up to early diakinesis as is the case in the human male.

The widely separated homologous chromosome regions devoid of chiasmata, which have been described at diakinesis in human spermatocytes after fixation for light microscopy (11, 15 ), are thus most likely a preparation artefact resulting from a general hypotonic swelling of the nuclei, denaturation of chromosomal proteins by acetic acid and alcohol traditionally used as fixatives and the airdrying procedure. 


\subsection{Terminalization of chiasmata}

The characterization of the crossover distribution (10) and the distribution of distinct synaptonemal complex segments at mid-late diplotene allows an assessment of whether and to what extent terminalization of chiasmata occurs (see 10 for a comparison between the crossover and the chiasma distribution for each of the autosomal bivalents). The comparison presented in Figure 30 allows the following conclusions: 1) In several respects, the distributions of synaptonemal complex segments at mid-late diplotene and the chiasmata share the same characteristics. In all bivalents, chiasmata and synaptonemal complex segments are frequent towards the telomere, whereas the centromeric chromatin and its flanking regions are nearly devoid of chiasmata and segments. Furthermore, chiasmata/synaptonemal complex segments are generally present in the middle of the long arms of bivalents 1-12 and in the short arms of bivalents 1-3, while chiasmata are more distal in the acrocentric bivalents 13-15. The long arms of bivalents 21-22 have one medial-subterminal peak while the short arms of the acrocentric bivalents generally are devoid of chiasmata as well as synaptonemal complex segments. 2) In all bivalents many more chiasmata than synaptonemal complex segments are present in the distal one-tenth of the bivalent arm, in particular in arms with only one chiasma (short arms of bivalents 3, 4-5, 9, 6-12, and both arms of bivalents 16-20), whereas the position of the medial chiasmata coincides with the position of synaptonemal complex segments.

This shows that terminalization of chiasmata does take place in diakinesis bivalents at least when the chiasmata are analyzed in preparations fixed and spread for light microscopy. If only one chiasma is present in the arm it is moved distally, whereas if two or more chiasmata are present only the distal one will move whereas the proximal ones remain at or close to their original position.

The mechanisms underlying these movements are at present not clear. The distribution of synaptonemal complex segments along the bivalents at early-mid diakinesis could not be analyzed in detail, but it appears very similar to that at mid-late diplotene and synaptonemal complex segments are generally absent in telomere regions. Hence, movement of chiasmata may not take place until the synaptonemal complex segments have been eliminated, i.e., for the majority of the chiasmata not until late diakinesis. In vivo chiasma terminalization during this stage might, however, be negligible as repulsion of homologous chromosomes appears to be absent in human spermatocytes and it is conceivable that the apparent distal movement of the chiasmata at late diakinesis in human spermatocytes is an artefact produced by conventional light microscopic methods which tend to separate the homologues.

According to these results, chiasmata are immobilized at their site of formation until the end of diakinesis whereafter terminalization may occur. This conclusion is supported by recent observations in grasshoppers (24), mouse (13) and Armenian hamster (1) where incorporation of bromodeoxyuridine at the penultimate $S$-phase, resulting in differential staining of substituted and unsubstituted chromatids, revealed that in these organisms chiasmata remain at their original position until diakinesis.

In Bombyx spermatocytes (7) and Coprinus meiocytes (8), chiasmata also appear to remain at the site of crossing over as evidenced by the constancy in the number of chiasmata until late diakinesis, i.e., the stage where the circular component of the chiasma is eliminated. Thereafter, terminalization may proceed and in agreement with this MAEDA (16) in his light microscopic studies reported a gradual terminalization of chiasmata during diakinesis in Bombyx spermatocytes until, by metaphase I, all chiasmata were present at the telomeres. These results suggest that the circular component of the chiasma plays a role in the immobilization of the chiasma.

The observation that terminalization of chiasmata towards the end of prophase may occur has a severe impact on light microscopic studies where information on the number and distribution of chiasmata is the objective. In several organisms, this is assessed by analyzing metaphase I bivalents, a stage where substantial movement of the chiasmata may have occurred. Furthermore, even analyses of earlier stages of diakinesis may be subjected to some error as early terminalization may occur in arms with only one chiasma. 
P.B. HOLM \& S.W. RASMUSSEN: Human meiosis VII
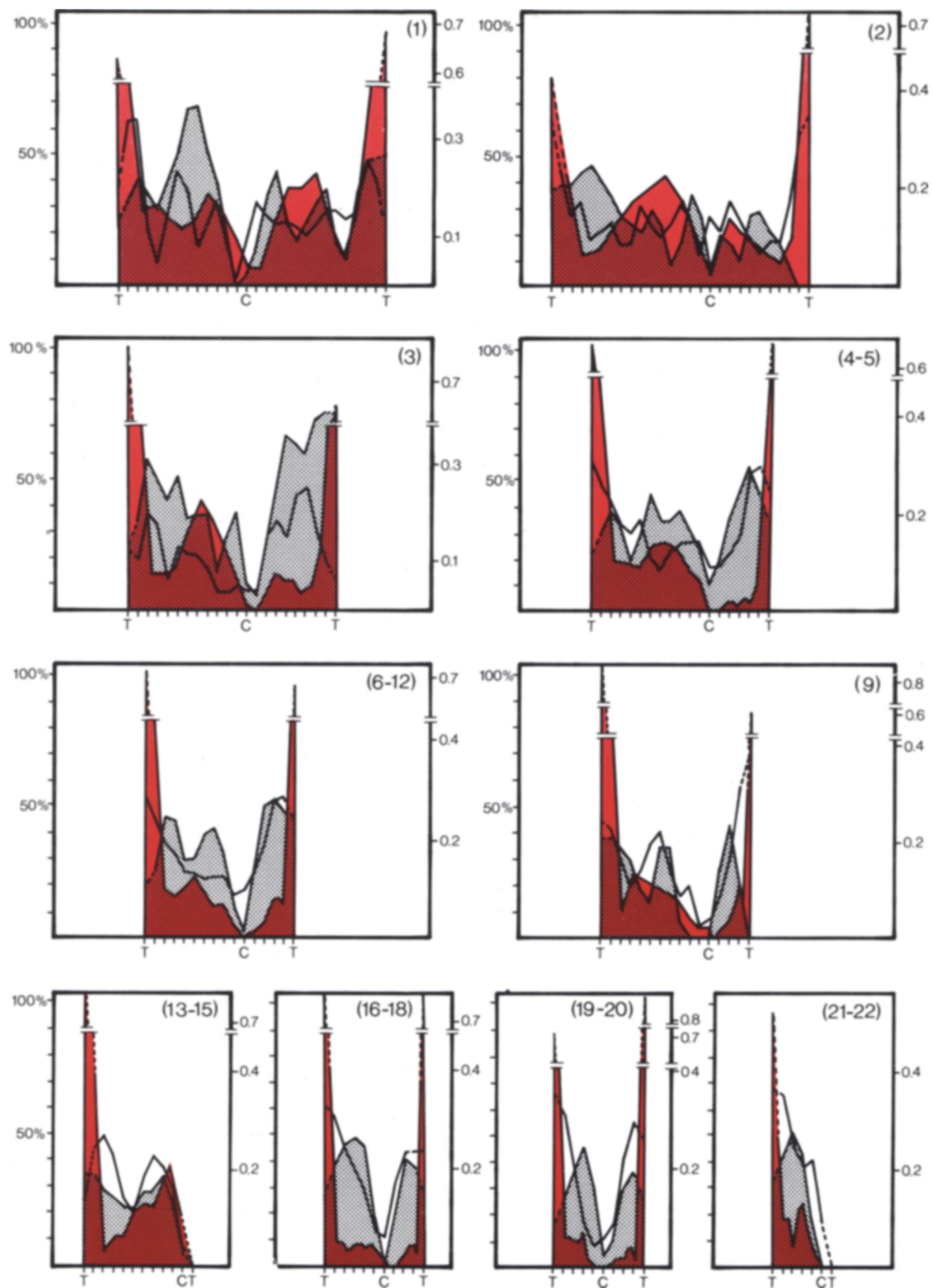
Figure 30. Frequency distribution of synaptonemal complex fragments along bivalents $1,2,3$, 4-5, 9, 6-12 (minus 9), 13-15, 16-18, 19-20 and 21-22 at mid-late diplotene (light hatching) compared to the distribution of crossovers (no hatching) obtained through the analysis of recombination nodules and bars at pachytene (10) and the distribution of chiasmata as seen in the light microscope at diakinesis (shown in red). The latter distribution is derived from the data published by Hultén (11). The left ordinate is the frequency of synaptonemal complex present and the right one the frequency of chiasmata and crossovers. The total frequency of crossovers had been corrected to match that of chiasmata, i.e., a mean of 50 per nucleus. As for distribution of crossovers, the chiasma frequencies shown are the mean of the frequency in each interval and a neighbour interval, see also legend to Figure 28.

\section{ACKNOWLEDGEMENTS}

It is a pleasure to thank D. von WeTTSTEIN for his constant support during the course of this work and for critically reviewing the manuscript. Expert technical assistance was provided by JEAN SAGE and the financial support by grant BIO-E$417 \mathrm{DK}(\mathrm{G})$ from the Commission of the European Communities and a Niels Bohr scholarship from the Royal Danish Academy of Sciences and Letters to Preben BaCh Holm.

\section{REFERENCES}

1. ALLEN, J.W.: BrdU-dye characterization of late replication and meiotic recombination in Armenian hamster cells. Chromosoma (Berl.) 74 , 189-207 (1979)

2. Berthelsen, J.G., P.B. Holm \& S.W. RASMUSSEN: Three ultrastructural markers on pachytene bivalents of human spermatocytes. Carlsberg Res. Commun. 45, 25-28 (1980)

3. Darlington, C.D.: Recent advances in cytology. 2nd ed. Churchill, London 671 pp (1937)

4. Gillies, C.B.: An ultrastructural analysis of chromosomal pairing in maize. Compt. Rend. Trav. Lab. Carlsberg 40, 135-161 (1975)

5. GiLlies, C.B.: The relationship between synaptonemal complexes, recombination nodules and crossing over in Neurospora crassa bivalents and translocation quadrivalents. Genetics 91, 1-17 (1979)

6. Holm, P.B. \& S.W. Rasmussen: Human meiosis I. The human pachytene karyotype analyzed by three dimensional reconstructions of the synaptonemal complex. Carlsberg Res. Commun. 42, 283-323 (1977)

7. Holm, P.B. \& S.W. Rasmussen: Chromosome pairing, recombination nodules and chiasma formation in diploid Bombyx males. Carlsberg Res. Commun. 45, 483-548 (1980)

8. Holm, P.B., S.W. Rasmussen, D. ZickleR, B.C. LU \& J. SAGE: Chromosome pairing, recombination nodules and chiasma formation in the basidiomycete Coprinus cinereus. Carlsberg Res. Commun. 46, 305-346 (1981)

9. Holm, P.B. \& S.W. Rasmussen: Human meiosis V. Substages of pachytene in human spermatogenesis. Carisberg Res. Commun. 48, 351-383 (1983)

10. Holm, P.B. \& S.W. Rasmussen: Human meiosis VI. Crossing over in human spermatocytes. Carlsberg Res. Commun. 48, 385413 (1983)

11. HultéN, M.: Chiasma distribution at diakinesis in the normal human male. Hereditas 76, 55-78 (1974)

12. JoHN, B. \& K.R. LewIS: The meiotic system. Protoplasmatologia, Vol. 6 Springer Verlag, Wien. (1965)

13. Kanda, N. \& H. Kato: Analysis of crossing over in mouse meiotic cells by BrdU labelling technique. Chromosoma (Berl.) 78, 113-121 (1980)

14. Kundu, S.C. \& Yu.F. Bogdanov: Ultrastructural studies of late meiotic prophase nuclei of spermatocytes of Ascaris suum. Chromosoma (Berl.) 70, 375-384 (1979)

15. Laurie, D.A., M. Hultén \& G. H. Jones: Chiasma frequency and distribution in a sample of human males: chromosomes 1,2 , and 9 . Cytogenet. Cell Genet. 31, 153-166 (1981)

16. MAEDA, T.: Chiasma studies in the silkworm, Bombyx mori L. Jap. J. Genet. 15, 118-127 (1939)

17. Polani, P.E., J.A. Crolla, M.J. Seller \& F. Molr: Meiotic crossing over exchange in the female mouse visualized by BudR substitution. Nature 278, 348-349 (1979)

18. Rasmussen, S.W. \& P.B. Holm: Human meiosis II. Chromosome pairing and recombination nodules in human spermatocytes. Carlsberg Res. Commun. 43, 275-327 (1978)

19. Rasmussen, S.W. \& P.B. Holm: Human meiosis IV. The elimination of synaptonemal complex fragments from metaphase I bivalents of human spermatocytes. Carlsberg Res. Commun. 43, 423-438 (1979)

20. Rasmussen, S.W. \& P.B. Holm: Mechanics of 
meiosis. Hereditas 93, 187-216 (1980)

21. SolarI, A.J.: The behaviour of chromosomal axes during diplotene in mouse spermatocytes. Chromosoma (Berl.) 31, 217-230 (1970)

22. Solari, A.J.: Chromosomal axes during and after diplotene. In: International Cell Biology 1980-1981, G. Schweiger ed., Springer Verlag, Berlin, Heidelberg \& New York, pp 178-186 (1981)

23. Solari, A.J. \& O. Vilar: Multiple complexes in human spermatocytes. Chromosoma (Berl.) 66, 331-340 (1978)

24. TEASE, C. \& G.H. Jones: Analysis of exchanges of differentially stained meiotic chromosomes of Locusta migratoria after BrdU-substitution and FPG staining. I. Crossover exchanges in monochiasmate bivalents. Chromosoma (Berl.) 69, 163-178 (1978)
25. Westergaard, M. \& D. von Wettstein: The meiotic cycle in an ascomycete. In: Effect of radiation on meiotic systems. Internat. Atom. Energy Agency. Panel Proc. Sci. Sti. Pub. 173, pp 113-121 (1968)

26. WestergaArd, M. \& D. von Wettstein: Studies on the mechanism of crossing-over IV. The molecular organization of the synaptinemal complex in Neottiella (Cooke) Saccardo (Ascomycetes). Compt. Rend. Trav. Lab. Carlsberg 37, 239-268 (1970)

27. ZICKLER, D.: Development of the synaptonemal complex and the "recombination nodules" during meiotic prophase in the seven bivalents of the fungus Sordaria macrospora Auersw. Chromosoma (Berl.) 61, 289-316 (1977) 